\title{
Fast Fully Adaptive Signalling for Target Matching
}

\author{
Colin P. Horne \\ Department of Electronic and Electrical Engineering, University College London, London, UK \\ uceehor@ucl.ac.uk \\ Aaron M. Jones \\ Air Force Research Laboratory, Wright-Patterson AFB, Dayton, USA \\ Graeme E. Smith \\ Department of Electrical and Computer Engineering, The Ohio State University, Columbus, USA \\ Hugh D. Griffiths \\ Department of Electronic and Electrical Engineering, University College London, London, UK
}

\begin{abstract}
In this article, we discuss a novel signal processing technique for adaptive radar that permits joint target-matched illumination and interference avoidance in dynamic spectral environments. This approach allows for spectral coexistence between a radar system and a primary user of the radio frequency space. Spectral coexistence is exploited to allow use of higher bandwidths than would otherwise be available to conventional radar systems. The technique proposed exploits the relative simplicity of the error reduction algorithm, and also provides a novel use of the masking procedure to allow for target-matched illumination. Practical constraints such as constant modulus are considered in the waveform design procedure, while providing an implied signal-to-interference-plus-noise ratio improvement via the error reduction algorithm. Results for full simulation and hardware-in-the-loop experiments are presented and analyzed. We are able to show a signal-to-interference-plus-noise ratio gain of $40 \mathrm{~dB}$ is achieved for the target-matched waveform as compared with a linear frequency modulated waveform. However, the signal-to-interference-plus-noise ratio gain comes at a cost of degraded autocorrelation characteristics of the targetmatched illumination waveform, despite only modest levels of primary user spectrum occupancy. Spectral notch depths achieved by the modified error reduction algorithm are approximately $25 \mathrm{~dB}$.
\end{abstract}

\section{INTRODUCTION}

The radio spectrum is a strictly limited resource, with highly regulated access leading to substantial competition for available space [1]-[4]. Bandwidth allocated exclusively to radar operations is under threat. It is being eroded in favour of alternative user communities, such as mobile communications. Sale of the rights to use any released spectrum will raise significant revenues [5][7], leading to further pressure on the radar community to relinquish frequency allocation. The electromagnetic environment in which future radar sensors will be required to operate will be 'congested, cluttered, contested, connected and constrained' [8]. The result of these combined influences will be a significant drop in performance for conventional radar systems operating in increasingly complicated environments with reduced resource availability.

In addition, small unmanned aircraft systems (SUAS) provide an attractive option as a sensor platform for operation in hostile environments, due to their relatively low cost, and the avoidance of hazardous environments for manned platforms. One disadvantage of SUAS is the restrictions placed on payload size and weight, and on available power (SWaP) within the airframe.

The challenge faced by radar designers is to provide systems with the required levels of performance given the likely hostile conditions, reductions in bandwidth, and SWaP constraints from deployment on SUAS platforms. To negate the loss of dedicated radar bandwidth, techniques can be employed to allow the radar to coexist with primary users (PU) of the radio spectrum [9], intelligently exploiting unused bandwidth, while complying with both access restrictions, and the conflicting requirements of high capability and SWaP.

We assume the spectral occupancy behaviours exhibited by the PU of the radio frequency (RF) space are dynamic, and potential targets manifest dynamic impulse responses. We propose the adoption of a fully adaptive radar (FAR), sometimes known as cognitive radar, solution [10]-[12] capable of adapting appropriate radar system operating parameters within the closed loop of a perception-action cycle (PAC) [10]. The PAC of a FAR provides the framework to observe environmental conditions, and tailor the system behaviour to provide improved performance by matching the operating characteristics to the perception of the environment and target. In this article we describe the Fast Fully Adaptive Signalling for Target matching (FFAST) algorithm. FFAST is a computationally lowcost and straightforward framework for radar waveform 
design. We implement functions within the context of the PAC, to achieve reduced levels of interference to the PU, and increased target signal-to-interference-plusnoise ratio (SINR), as compared with a fixed radar signal. This is achieved through the exploitation of the increased availability of bandwidth, currently unused by the PU. Finer range resolution is achieved and, with that, extended target responses that provide the possibility to match the illumination signal to the target.

This strategy produces a dynamically adaptive solution capable of operating in non-stationary, spectrally congested environments. As we will show the SINR is improved compared with a linear frequency modulated (LFM) waveform. We consider a single target whose range profile response evolves over time. Preliminary results were first reported in [13]. The approach taken trades some level of performance against a reduced computational load, with the aim of power and weight savings and reducing the computational burden of the adaptive algorithm. We accomplish this while considering the practical waveform design constraints of limited bandwidth and constant modulus. The negative impact of the waveform design on the ambiguity function are illustrated, but solutions are not addressed.

Straightforward models of the PU transmissions and target responses are utilised to ensure adequate reproduction of the signals when implementing the framework on an experimental hardware platform. This provides for repeatability and consistency between the simulation and over-the-air results.

The scenario described in this work relies on the application of waveform diversity, [14]-[17] in two respects. Firstly in the form of spectral coexistence, aimed at allowing multiple RF systems to operate in the same geographical locality and spectral region. Secondly in the design of transmit waveforms matched to the target response to achieve an increase in SNR. Haykin et al. [18] recognise the connection between the perceptionaction cycle found in cognitive radar and optimal waveform design based on matching the waveform to some characteristic of the sensed environment on an on-going basis. This has become a central theme explored in cognitive radar research.

Waveform design has attracted interest over a long period. Bell [19] considers waveform and filter design for optimum detection of extended targets, and contrasts the result with a mutual information design scheme for information extraction. Setlur et al. [20] expand on the mutual information approach taken by Bell to create a two transmission epoch design process which results in an improved distribution of the available energy. In [21] the authors address the problem of waveform design in the context of a spectrally crowded environment. Selesnick et al. [22], [23] describe iterative algorithms for creating notched and multiple notched chirp-like waveforms allowing transmissions on selected frequencies to be controlled. Spectral notching in FM noise radar in the presence of narrowband noise is analysed in [24], and experimentally demonstrated in [25]. Aubry et al. [26] employ an optimisation based approach to waveform design in the spectral coexistence context, the perception element of the cognitive process relying on external environment knowledge provided by a radio environment map (REM). The authors provide a useful summary of the merits and drawbacks of various optimisation based waveform design approaches. Huang et al. [27] introduce the notion of bandwidth quality to the coexistence problem, again using a REM to provide the cognitive sensing element of the architecture.

The performance cost of introducing spectrally disjoint radar waveforms is considered in [28], and in [29] the impact of spectral notching on beamforming performance in phased array architectures is assessed.

The principles of waveform design for target-matched illumination (TMI) are described by Gjessing [30]. The authors in [31] derive performance bounds for the optimisation of transmit waveform, channel, target and receiver. In [32] specifically designed radar waveforms are compared with LFM waveforms for target detection and discrimination. Orthogonal frequency division multiplexing waveform design is examined in [33] for adapting to an extended target response and non-stationary interference, which maximises the mutual information between the target response and the received signal. The joint implementation of spectral coexistence with TMI is investigated in [34] for the maximization of target SINR while adhering to external spectral costraints, and [35] undertakes the design of transmit waveforms by considering the transmit signal - target mutual information in a spectral coexistence context. The use of TMI, in addition to being applied to improve target detection, is also applied to the target identification objective [36][39].

Aubry et al. [40] extend the scenario described in [21] to include signal dependent interference. Zhang et al. [41] consider the joint transmit waveform and associated receive filter design in signal dependent interference, employing an iterative algorithm to maximise the target SINR. The development of a practical testbed is described in [42] and used to experimentally demonstrate an improvement in signal to clutter ratio given an $a$ priori estimate of target response, in addition interference avoidance is addressed given a priori environmental spectral knowledge. In [43] and [44] knowledge of the target SNR and mutual information are used to match the designed waveform to targets in cluttered environments.

Many other works address waveform design under various constraints including [45]-[51]. The design of a cognitive radar architecture with application to the problem of matched illumination is described in [52]. 
In order to design suitable waveforms for spectral coexistance, the environmental conditions must be known or estimated. The mechanisms to attain knowledge of spectral and temporal behaviour of RF radiators can be static, derived from information defining fixed frequencies and bandwidths of PUs such as radio stations, or a priori knowledge of dynamic PU behaviour. For less predictable radiators, a REM [21], [40] can supply live information regarding RF behaviour in the environment. For our scenario, operating on a remote, self contained sensor platform, in an unknown spectral environment, assessment of third party transmissions will be from continuous or periodic monitoring of the environment, [53], [54], providing a live description of the prevailing conditions, without the necessity for third-party involvement. Alternative advanced spectrum sensing techniques might also be applied [55], [56].

The remainder of this article is structured as follows. Section II addresses the formulation of the problem of interference avoidance and TMI. The proposed solution is presented in Section III. Section IV describes the hardware and software frameworks employed in the experimentation. Section V describes the results obtained. Section VI completes the paper with conclusions.

The notation used in this work is as follows. We denote column vectors as lower-case, bold-face letters. The $n^{t h}$ entry of a column vector $\mathbf{s}$ is characterised $\mathbf{s}_{\mathbf{n}}$. Matrices are shown using boldface upper-case letters. Scalar quantities are denoted by upper-case letters. The Hermitian operator is given as $(\cdot)^{H}$, while the Fourier transform and inverse Fourier transform are represented as $\mathscr{F}$ and $\mathscr{F}^{-1}$ respectively. The Hadamard operation is given by the $\odot$ symbol, the convolution operation is $*$, correlation is represented by $\star$, and $\angle$ is the angle operator. Finally, the absolute value is denoted by $|\cdot|$, and the vector norm by $\|\cdot\|$.

\section{Problem Formulation}

Consider a scenario in which a monostatic radar is operating in a dynamic, congested spectral environment, where the radar is a secondary user of the RF space. A single static target which exhibits a dynamic range profile over time exists within the scene. The goal is to observe the environment, and use the perception of current conditions to design a waveform which avoids transmitting unacceptably high levels of power at the instantaneous frequencies occupied by the PU. Further, the frequency restricted waveform is used to probe the dynamic target, such that a new waveform, jointly optimised for both PU avoidance and target matched illumination is designed. The process exists as part of an ongoing perception-action cycle, whereby the target probe and environmental observation are repeated at intervals such that the system adapts to the dynamic behaviours of target and PU. Signal dependent interference, or clutter, could also be estimated from data derived from range cells neighbouring the target, however, clutter processing is not considered in this work.

Consider the continuous time, pulsed radar waveform, $s_{\mathrm{RF}}(t)$, which is non-zero over $\mathrm{t} \in[0, T]$ for pulse duration $T$ :

$$
s_{\mathrm{RF}}(t)=\alpha(t) \exp \left\{j\left(\omega_{\mathrm{c}} t+\phi(t)\right)\right\}
$$

where $\alpha(t)$ is the pulse envelope, $\omega_{\mathrm{c}}$ the carrier frequency in radians per second and $\phi(t)$ the waveform phase, measured in radians.

The equivalent baseband waveform can be expressed as:

$$
s(t)=\alpha(t) \exp \{j \phi(t)\}
$$

Critically sampling the baseband transmit waveform, we can represent the signal in a complex-valued vector form, $\mathbf{s} \in \mathbb{C}_{\mathrm{M} \times 1}$,

$$
\mathbf{s}=\boldsymbol{\alpha} \odot \exp \{j \boldsymbol{\phi}\}
$$

where $\boldsymbol{\alpha}$ is the amplitude vector of the baseband waveform, $\phi$ the phase components, and $M$ the number of samples within the pulse length.

The signal, $\mathbf{r}$, received by the radar in response to the transmit signal, s, interacting with the target, along with the PU interference signal and noise, collected from each processing interval can be represented by:

$$
\mathbf{r}=\mathbf{s} * \mathbf{w}+\mathbf{i}+\mathbf{n}
$$

where $\mathbf{w}$ is the range profile response of the target, $\mathbf{i}$ is the PU interference, and $\mathbf{n} \in \mathbb{C N}\left(0, \sigma^{2}\right)$ represents the additive white Gaussian noise. The target range is assumed known allowing the collection of returns to be limited to only the vicinity of the target location.

The received signal, $\mathbf{r}$, is correlated with the transmitted signal, s, resulting in the filtered receive signal $\mathbf{r}_{\mathrm{f}}$ which could be the input to a detection process, but here provides for SINR estimation :

$$
\mathbf{r}_{\mathrm{f}}=\mathbf{s} \star \mathbf{r}
$$

The problem to be addressed is, given the signal from the radar receiver $\mathbf{r}$, how should we design the waveform, $\mathbf{s}$, to be transmitted during the following coherent processing interval (CPI) to achieve improvement in SINR? Our proposed solution is discussed in Section III, but first we describe the modelling of the PU interference signal, $\mathbf{i}$, and the target response, $\mathbf{w}$. The CPI consists of the period over which a single transmit waveform is employed. In this work all data collection and processing occurs over the period of a single CPI.

The PU emulation waveform, considered as interference to the radar, is implemented through a straightforward signal model consisting of the sum of multiple 
non-overlapping, increasing frequency, LFM waveforms. Although this is not a realistic model of PU behaviour, it provides for reproducibility in the hardware test platform. The PU is made dynamic by the addition of two different signal model instantiations, each consisting of a number of LFMs with selected frequencies and bandwidths. The first model instantiation represents the PU occupancy at the start of the experiment which begins at its maximum amplitude and decreases in amplitude over the course of the experiment. The second instantiation is the PU occupancy at the termination of the experiment, which starts at zero amplitude and increases to its maximum level over the experiment.

Defining $\mathbf{n}=[0,1,2 \ldots(M-1)]$ as the vector of sample number within the signal, and $f_{\mathrm{s}}$ the sampling frequency, $\mathbf{t}_{\mathrm{s}}=\mathbf{n} / f_{\mathrm{s}}$, the time sample vector, each LFM is defined in discrete form as:

$\mathbf{l}\left(\mathbf{t}_{\mathrm{s}}, f_{0}, B, T\right)=\left\{\begin{array}{l}\exp \left\{j 2 \pi\left(f_{0} \mathbf{t}_{\mathrm{s}}+\frac{B}{2 T} \mathbf{t}_{\mathrm{s}}^{2}\right)\right\}, 0 \leqslant t \leqslant T \\ 0, \text { otherwise }\end{array}\right.$

where $f_{0}$ is the initial LFM frequency, $B$ the bandwidth, and $\mathrm{T}$ the duration of the LFM sweep, equal to the pulse extent.

The total PU evolution at time step $k$, denoted $\mathbf{i}_{\mathrm{k}}$, is therefore represented by:

$$
\begin{aligned}
\mathbf{i}_{\mathrm{k}} & =\alpha_{\mathrm{k}} \sum_{u=1}^{U} a_{\mathrm{I}_{\mathrm{u}}} l\left(\mathbf{t}_{\mathrm{s}}, f_{\mathrm{I}_{\mathrm{u}}}, B_{\mathrm{I}_{\mathrm{u}}}, T\right) \\
& +\beta_{\mathrm{k}} \sum_{v=1}^{V} a_{\mathrm{F}_{\mathrm{v}}} l\left(\mathbf{t}_{\mathrm{s}}, f_{\mathrm{F}_{\mathrm{v}}}, B_{\mathrm{F}_{\mathrm{v}}}, T\right)
\end{aligned}
$$

subject to the following, assuming $f_{\mathrm{I}}$ and $f_{\mathrm{F}}$ represent ascending ordered lists of LFM start frequencies, to enforce the non-overlapping LFM constraint:

$$
\begin{gathered}
f_{\mathrm{I}_{\mathrm{u}}}-f_{\mathrm{I}_{\mathrm{u}-1}} \geq B_{\mathrm{I}_{\mathrm{u}-1}}, \forall u \in\{2 . . U\} \\
f_{\mathrm{F}_{\mathrm{v}}}-f_{\mathrm{F}_{\mathrm{v}-1}} \geq B_{\mathrm{F}_{\mathrm{v}-1}}, \forall v \in\{2 . . V\}
\end{gathered}
$$

where $\alpha_{\mathrm{k}}$ and $\beta_{\mathrm{k}}$ represent linear increasing and decreasing factors respectively applied to the each PU model instantiation over the duration of the experiment and given by :

$$
\begin{aligned}
& \alpha_{\mathrm{k}}= \begin{cases}1-\frac{k}{K_{\mathrm{sim}}}, & \text { if } 0 \leqslant k \leqslant K_{\text {sim }} \\
0, & \text { otherwise }\end{cases} \\
& \beta_{\mathrm{k}}= \begin{cases}\frac{k}{K_{\text {sim }}}, & \text { if } 0 \leqslant k \leqslant K_{\text {sim }} \\
0, & \text { otherwise }\end{cases}
\end{aligned}
$$

$k$ and $K_{\text {sim }}$ are the current CPI number, and total number of CPIs in the experiment duration respectively. The value of $k$ starts at zero and increments by one after each processing interval to a maximum value of $K_{\text {sim }}-1$. Further, Equation (7) consists of the summation of two signals, each the addition of the number of LFM components, $U$ and $V$, used to construct the initial and final PU signal model instantiations respectively. Subscripts $I$ and $F$ indicate either initial or final PU model parameters respectively. The factor $a$, subject to the above subscripts, is the amplitude of the LFM component. The frequency characteristics of the PU are assumed to be constant over the period of one CPI.

The target response, $\mathbf{w}_{\mathrm{k}}$, is modelled by similar means to the PU interference. The target evolution can be represented by:

$$
\begin{aligned}
\mathbf{w}_{\mathrm{k}} & =\alpha_{\mathrm{k}} \sum_{x=1}^{X} a_{\mathrm{I}_{\mathrm{x}}} l\left(\mathbf{t}_{\mathrm{s}}, f_{\mathrm{I}_{\mathrm{x}}}, B_{\mathrm{I}_{\mathrm{x}}}, T\right) \\
& +\beta_{\mathrm{k}} \sum_{y=1}^{Y} a_{\mathrm{F}_{\mathrm{y}}} l\left(\mathbf{t}_{\mathrm{s}}, f_{\mathrm{F}_{\mathrm{y}}}, B_{\mathrm{F}_{\mathrm{y}}}, T\right)
\end{aligned}
$$

subject to the following:

$$
\begin{gathered}
f_{\mathrm{I}_{\mathrm{x}}}-f_{\mathrm{I}_{\mathrm{x}-1}} \geq B_{\mathrm{I}_{\mathrm{x}-1}}, \forall x \in\{2 . . X\} \\
f_{\mathrm{F}_{\mathrm{y}}}-f_{\mathrm{F}_{\mathrm{y}-1}} \geq B_{\mathrm{F}_{\mathrm{y}-1}}, \forall y \in\{2 . . Y\}
\end{gathered}
$$

where $\alpha_{\mathrm{k}}$ and $\beta_{\mathrm{k}}$ are defined by (8) and (9) respectively. $X$ and $Y$ represent the number of LFM components in the initial and final target response model instantiations. Subscripts $I$ and $F$ represent initial and final values of LFM parameters. The frequency characteristics of the target are assumed to be constant over the period of one CPI. As for the PU model described earlier, this model does not represent a realistic target response, but provides a straightforward and reproducible solution for the early assessment of the framework.

\section{PROpOSED SOLUTION}

In this section we describe the FFAST framework, and specific algorithms employed to implement elements of the solution.

The FFAST algorithm provides a fully adaptive radar solution by implementing a multi-loop PAC framework. An outline of the framework is provided in Algorithm 1. Our proposed solution, while certainly not unique, includes the basic structure consisting of three sequential loops, two PACs and one non-PAC loop as follows:

- Environment Sensing PAC (ESPAC)

- Target Interrogation PAC (TIPAC)

- Exploitation cycle (EC)

The ESPAC exists to sense, and adapt to, the background environment. The perception element of the PAC consists of collecting signals from the radar receiver over a period of a CPI, and creating an estimated occupancy mask of the frequencies at which the PU is currently transmitting. The data collection is carried out with the local radar transmission inactive to avoid 
RF contamination. The action component of this PAC is the design of an initial target interrogation waveform, $\mathrm{S}_{\mathrm{INT}}$, containing notches at the locations of estimated PU occupancy, to avoid causing interference to the PU, for use in the second PAC. Ideally the environmental sensing process is repeated at intervals matched to the dynamic behaviour of the PU such that the required response to PU occupancy changes is achieved. In this work constant update intervals are used.

The second algorithm phase, called TIPAC, instigates the transmission of a series of pulses of the $\mathrm{s}_{\text {INT }}$ waveform, collecting radar returns from the target, plus PU transmissions and noise over a CPI. The perception gained from this process consists of a description of the target estimated frequency response, outside of current PU occupied frequencies. The action is to design a waveform, $\mathbf{s}_{\mathrm{TMI}}$, which both avoids the PU occupancy, and matches the signal to the target characteristics by combining the previously estimated PU occupancy with the estimated target response to form a composite frequency mask. The TIPAC should repeat at intervals in some way matched to the target response dynamic behaviour. In practice we use constant update intervals.

The final, non-PAC, loop is the EC, which exploits the waveform designed from the previous phases, over multiple CPIs, to provide improved SINR for the target, compared with a non adaptive system, while still avoiding the PU occupancy. No further adaptation currently takes place in this phase.

As noted the nature of the dynamics of the PU frequency occupancy and target response defines the required repetition interval for the PACs. In the current framework constant repeat intervals are chosen, although adaptive control based on performance metrics could be included.

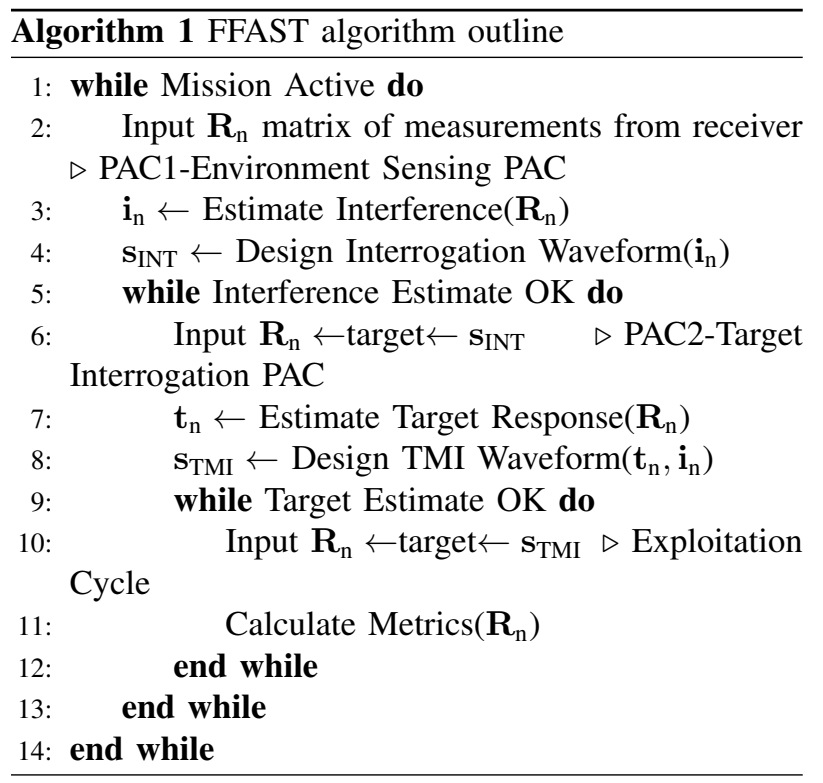

The algorithms employed in the estimation of the RF environment, and the waveform design process are described in the following sections.

\section{A. PU Environment estimation}

Under the assumption of Gaussian distributed signals, estimation of the spectral occupancy of the PU is achieved by making use of the Minimum Description Length (MDL) algorithm [57] to evaluated the number of interference signals present in the sampled data.

The environmental estimate enables the creation of a spectral, or Fourier Transform mask (FTM), which provides the waveform design constraints in the Fourier domain.

From [57], the MDL is evaluated as:

$$
\begin{aligned}
M D L(k)= & -\log \left(\frac{\prod_{i=k+1}^{p} l_{\mathrm{i}}^{\frac{1}{p-k}}}{\sum_{i=1}^{p} l_{\mathrm{i}}}\right)^{(p-k) N} \\
& +\frac{1}{2} k(2 p-k) \log (N)
\end{aligned}
$$

where $\mathrm{N}$ is the number of observations, $\mathrm{p}$ is the number of samples in each observation, $k$ is the number of signals being modelled, and $l$ represents ordered estimates of the eigenvalues of the sample covariance matrix, formed from the sampled interference and noise data.

The $M D L(k)$ value given by Equation (11) is evaluated for each value of $k$. The estimate for the number of signals contained in the sampled waveform is given by the value of $k$ for which the $M D L(k)$ is minimised. The $k$ largest values from the average estimated power spectral density (PSD) calculated over $N$ observations are taken as the frequency components containing the interference signals. The allowable spectral occupation for the radar waveform can then be formulated as the FTM based on these components, $\mathbf{q}=\left[q_{1}, q_{2} \ldots q_{\mathrm{k}}\right]$, where $q_{\mathrm{i}} \in[0,1], 0$ and 1 representing estimated PU occupied frequency bins, and noise-only frequency bins respectively.

An example interference PSD and the associated estimated binary signal mask, created by the application of (11) are shown in Fig. 1. The plot represents an instantaneous snapshot of the dynamic environment. In this example the two interference peaks are defined with equal bandwidths. The second peak, and therefore the associated masked region is seen to be wider than the first peak, this is due to leakage of energy into adjacent frequency bins in the FFT processing. The use of longer, zero filled, FFTs removes the issue. Alternative rank estimation techniques could be applied in place of the MDL [58], [59].

\section{B. Waveform design}

Phase-only transmit signal design allows for the creation of a waveform with approximately the required 


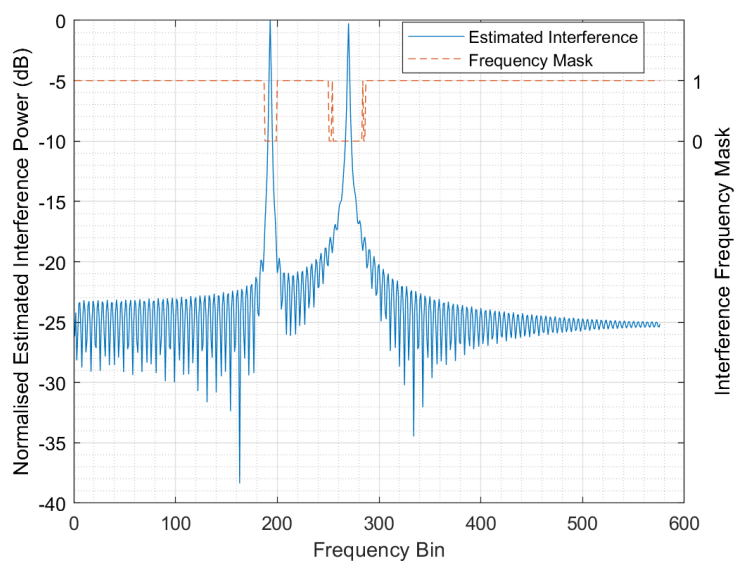

Fig. 1: Example interference (ESPAC) estimate and corresponding frequency mask computed with rank estimate provided by MDL for length 576 baseband signal

spectral character keeping the modulus of the signal constant.

Error Reduction Algorithm (ERA) [60], [61] is used as an efficient design technique to shape the spectrum while meeting the phase-only constraint. The algorithm, described previously in many references, is repeated here for convenience in Algorithm 2.

In our scenario, the frequency domain constraints from the environment sensing phase, require that nulls be placed at the locations defined by the FTM to create the target interrogation waveform, $\mathbf{S}_{\mathrm{INT}}$. Secondly, these nulls are overlaid with the target frequency domain characteristics from the target interrogation to create the TMI waveform $\mathbf{s}_{\mathrm{TMI}}$. In this work the algorithm is initialised with a constant modulus LFM waveform (6).

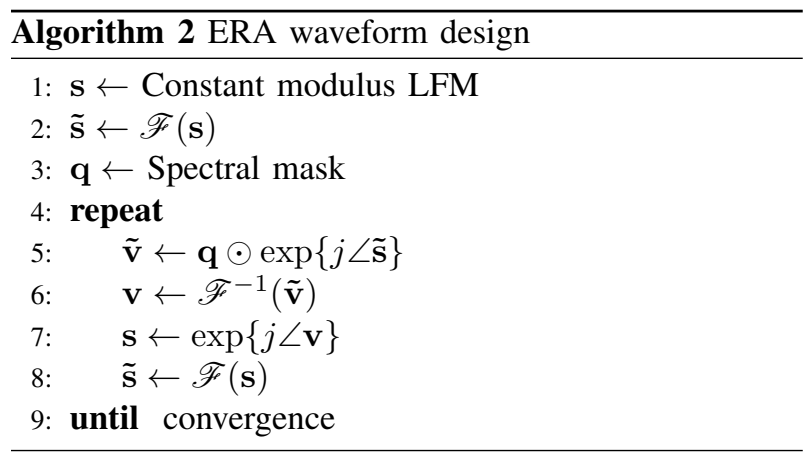

The ERA iteratively computes a new waveform while alternately meeting the constraints in the Fourier (spectral mask) and time (modulus) domain. The design process terminates when the convergence criterion is met. Convergence is assumed if the difference between the squared errors between the designed waveform and
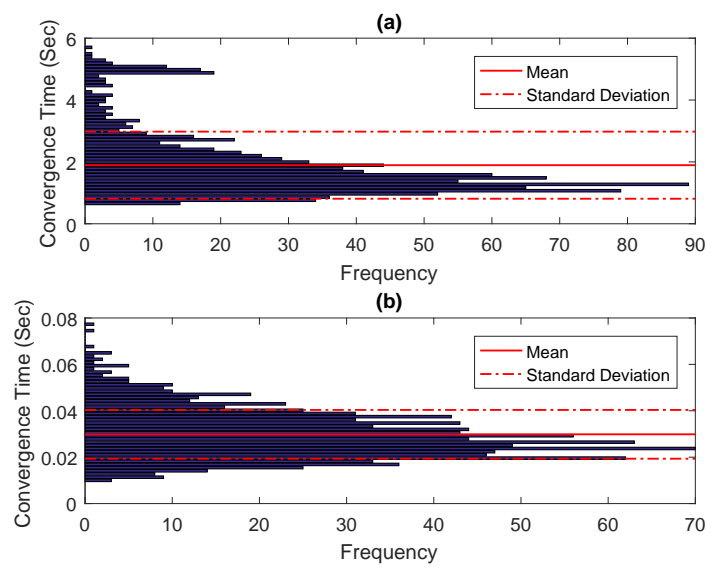

Fig. 2: Waveform design convergence time comparison (a) Quasi-Newton method and (b) ERA, showing more than an order of magnitude lower mean convergence time for the ERA in this scenario

the specification, on two successive passes, is within $1 \times 10^{-6}$.

A statistical analysis of the convergence time for the ERA compared against the quasi-Newton method motivates continued use of the ERA. The analysis is based on the computational cost of solving a similarity constraints waveform design problem [62], [63] using Monte-Carlo simulation. The optimisation is formulated to create the waveform $\mathbf{s}$ as :

$$
\min _{\varphi}\left(\sum|| \tilde{\mathbf{s}}(\varphi)|-\mathbf{q}|^{2}\right) \quad \text { subject to }|\mathbf{s}|=1
$$

where $\mathbf{s}$ is initialised with a constant modulus, random phase waveform, $\tilde{\mathbf{s}}=\mathscr{F}(\mathbf{s})$, and $\tilde{\mathbf{q}}$ is the required waveform spectral mask specification.

The optimisation is implemented using the Matlab ${ }^{\circledR}$ unconstrained optimisation function 'fminunc'. To ensure a fair comparison with the ERA results, an additional constraint is added to the objective function in the fminunc formulation. This constraint allows the minimisation process to be considered as complete should the error achieved be equal to, or less than, that achieved by the ERA solution.

Results from this comparison are illustrated in Fig. 2, for 1000 iterations of the Monte-Carlo simulation. The measured times are derived from the Matlab ${ }^{\circledR}$ 'tic' and 'toc' functions. Observed convergence times for the similarity waveform optimisation have a mean $1.9 \mathrm{~s}$, while the ERA is approximately $0.03 \mathrm{~s}$. Approximately $5 \%$ of the similarity solution iterations did not achieve the convergence criteria within the 120,000 function calls set as the upper limit.

As the most computationally costly aspect of the ERA is the calculation of a forward and reverse FFT pair, the 
practical implementation of the ERA for deployment on SUAS will benefit from the efficient implementation of FFTs in field programmable gate arrays (FPGA).

\section{Target response estimation}

The waveform designed from the environmental estimation phase is transmitted to interrogate the extended target response. Returns collected from a number of transmissions of the pulsed waveform are processed in a similar manner to the PU evaluation data using the MDL procedure from III-A above. The procedure diverges from the PU estimation in the formation of the FTM. Whereas the PU processing creates $q_{\mathrm{i}} \in\{0,1\}$, the target response estimation creates $q_{\mathrm{i}} \in[0,1]$ corresponding to the normalised PSD of the $k$ largest PSD estimates. This new FTM will include frequency components from the PU transmissions in addition to the required target response. To ensure the FTM is restricted to the estimated target response it is scaled by the FTM from the PU estimation process which eliminates the contributions from the PU:

$$
q_{\mathrm{TMI}}=q_{\mathrm{TARGET}} \odot q_{\mathrm{PU}}
$$

where $q_{\mathrm{TMI}}$ is the FTM required for creating the TMI waveform, $q_{\text {TARGET }}$ is the FTM created by the target response estimation and $q_{\mathrm{PU}}$ is the FTM created from PU estimation.

\section{TMI waveform design}

The TMI waveform design follows precisely the procedure described in III-B above. The use of the composite FTM from III-C enables the ERA to create the required waveform.

\section{Simulation AND EXPERIMENTAL CONFIGURATION}

A common framework for simulation and hardwarein-the-loop (HITL) experimentation has been developed in Matlab ${ }^{\circledR}$ running under the Windows ${ }^{\circledR}$ operating system. The framework start-up parameters dictate whether transmitter, environment, target, receiver interactions are achieved by simulation, or are carried out over-the-air using hardware transmitters and receivers. The functionality is abstracted such that consciousness of the mode of operation is restricted to the interface functions which either communicate with, or simulate the hardware components. In simulation mode no attempt is made to simulate the over-the-air path losses between the hardware components, or distortion effects introduced by the transmitter and receiver chains. These effects could easily be added to the simulation components in the future. However, delays due to the cable length connecting the system rack to the radar heads are included such

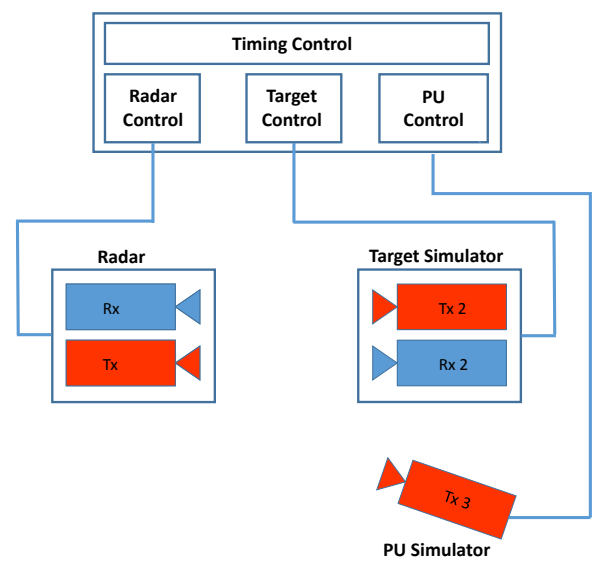

Fig. 3: Physical layout representation for joint spectral coexistence and TMI experiments

that range delay measurements are common between simulation and HITL operation.

Fig. 3 illustrates the components of the simulation/HITL framework. The Timing Control system is responsible for coordinating all activities. The remaining three control blocks implement the control and processing activities required by the radar, target and PU simulation components.

It can be seen that the target simulator is represented as a transmitter-receiver pair. In both full simulation and HITL modes no physical target is employed. Instead, the target is either fully simulated in software, or in HITL, by receiving the over-the-air radar transmission in a separate receiver, convolving this signal with the current target response in software, and re-transmitting the results overthe-air for reception by the radar. This allows for flexible, repeatable control of the target behaviour across both the full simulation and HITL experiments.

Details of the dynamic PU emulation, and the dynamic target response employed in the target simulator are described in section II.

The mapping of the framework from pure simulation to HITL requires a hardware solution providing highly flexible, highly capable functionality. To provide this capability, the framework is designed around the use of the Cognitive Radar Experimental Workspace (CREW) [64] at The Ohio State University ElectroScience Laboratory. However, by replacing the components specifically related to interfacing with the CREW hardware, it is equally applicable to alternative hardware platforms.

The CREW system consists of four transmitting channels plus four receiving channels, each operating at $94 \mathrm{GHz}$, with an instantaneous bandwidth of $1 \mathrm{GHz}$. Real value sampling is carried out at $3 \mathrm{GHz}$, and the Hilbert Transform applied to create complex samples 


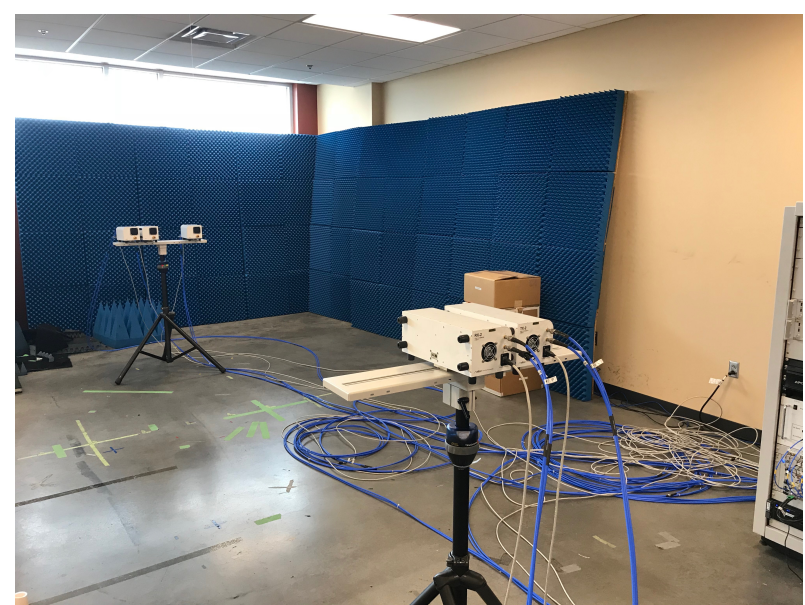

Fig. 4: CREW Monostatic radar (foreground), target and PU simulators (background). The blue phased-matched cables connect the transmit and receive heads to the main control rack

over $1.5 \mathrm{GHz}$ with a usable bandwidth of $1 \mathrm{GHz}$. Each of the channels has its own transmitting or receiving head, containing the final up/down RF conversion stages, connected to the main system rack by cables, allowing geographically diverse transmit/receive scenarios to be constructed under laboratory conditions. The main system rack contains four channels of arbitrary waveform generation and digitisers. Control of the system is provided by an integrated personal computer, running the Windows ${ }^{\circledR}$ operating system, and Matlab ${ }^{\circledR}$ software. Algorithms to create waveforms, process received data, and any other processing requirements, are run within the Matlab ${ }^{\circledR}$ environment, which has full access to the hardware subsystems, enabling real-time control of the entire system.

The radar sensor in the HITL experiments consists of a CREW receiver/transmitter pair. The target simulator is formed from a second receiver/transmitter pair. The interference is generated in a third CREW transmitter. The physical layout is shown in Figure 4, with target simulator receiver and transmitter, along with the interference simulation transmitter seen at the far end of the laboratory. The two heads seen in the foreground form the primary radar.

The detailed sequence of events which takes place over the course of the experiment are illustrated by the flow chart in Fig. 5. As previously described, the environment sensing phase and target interrogation phase are both PACs, where actions are taken based on the perception of current conditions. Each cycle of the PACs and EC persists for the duration of multiple transmit pulses constituting a complete CPI, providing multiple observations of the environment or target. The PU gener-

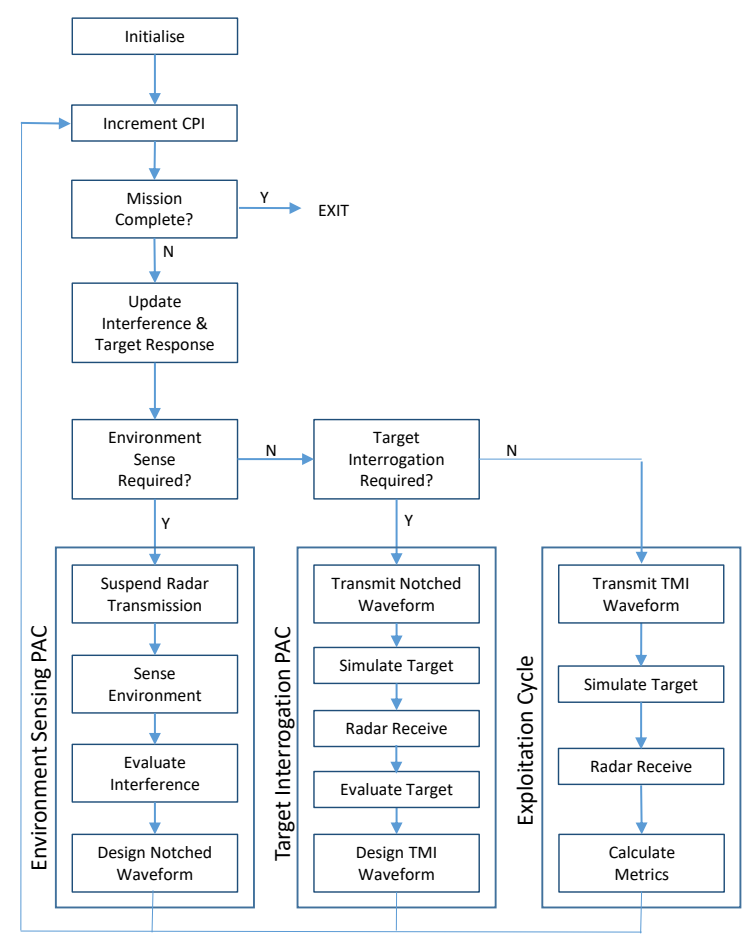

Fig. 5: Flow chart representation of the FFAST framework

ated interference, and the target response are updated on each new CPI. Both are pre-calculated prior to the experiment. As previously stated the environmental observation interval, and the target interrogation interval are both predefined in the current experiments. If the PU observation time has arrived, as detected by the 'Environment Sense Required?' block based on the number of processing intervals since the previous assessment, the radar transmitter is switched off, the environment assessed to create an estimate of PU occupancy, and the new target interrogation waveform designed. Always following an environment assessment, and possibly more frequently as assessed by the 'Target interrogation Required?' block based, as above, on the number of intervals since the previous assessment, the target interrogation phase takes place. The target interrogation phase is responsible for estimating the latest extended target response by employing the previously designed interrogation waveform. From the resulting data an updated TMI waveform is designed. During all remaining CPIs, the TMI waveform is employed to detect the target with SINR and other performance metrics being assessed. This is known as the exploitation cycle.

Due to the high processing loads imposed by simulation oriented tasks, particularly transfer of data to/from 
the personal computer, and target simulation processing, the experimental framework does not run in real time. A stop-frame approach is taken whereby each function is initiated, such as radar transmission, and all functions which depend on that, for example target simulator reception, are synchronised such that they gather the correctly timed real-time data. The most significant bottleneck to real-time operation however, is caused by the archiving of data to hard disk. All data collected by the radar and target simulator receivers, in addition to control data, and waveform designs, is stored for later off-line analysis. Real-time operation would be achievable if the algorithms were implemented in hardware close to the digital interfaces, a physical target replaces the target simulation, and data archiving suspended.

The framework produces various performance assessment graphs during experiments, examples of which can be seen in Section V.

TABLE I: Radar operating parameters

\begin{tabular}{|c|c|}
\hline Parameter & Value \\
\hline Experiment centre frequency & $94.28 \mathrm{GHz}$ \\
\hline Tx/Rx bandwidth & $1 \mathrm{GHz}$ \\
\hline Max. Tx power & $25 \mathrm{dBm}$ \\
\hline Antenna gain & $33 \mathrm{~dB}$ \\
\hline Antenna beamwidth (Az) & $9^{\circ}$ \\
\hline Antenna beamwidth (El) & $11^{\circ}$ \\
\hline Sample frequency & $3 \mathrm{GHz}$ \\
\hline Sample type & $12 \mathrm{bit}, \mathrm{Real}$ \\
\hline IF & $800 \mathrm{MHz}$ \\
\hline Operating band & $300-1300 \mathrm{MHz}$ \\
\hline Transmit pulse length & $10 \mathrm{kHz}$ \\
\hline PRF & 128 \\
\hline Pulses per CPI & $5 \mathrm{CPIs}$ \\
\hline Listen interval & $5 \mathrm{CPIs}$ \\
\hline Target interrogation interval & $200 \mathrm{CPIs}$ \\
\hline Experiment length & $1152 \mathrm{samples})$ \\
\hline
\end{tabular}

TABLE II: Interference and target parameters

\begin{tabular}{|c|c|}
\hline Parameter & Value \\
\hline Interference update interval & $1 \mathrm{CPI}$ \\
\hline Number of LFMs in interference $-Q_{\text {START }}$ & 2 \\
\hline Interference start LFM base frequencies - $f_{0}$ & $497,697 \mathrm{MHz}$ \\
\hline Interference start LFM bandwidths - $B$ & $6,6 \mathrm{MHz}$ \\
\hline Number of LFMs in interference $-Q_{\mathrm{END}}$ & 1 \\
\hline Interference end LFM base frequency $-f_{0}$ & $1194 \mathrm{MHz}$ \\
\hline Interference end LFM bandwidth $-B$ & $12 \mathrm{MHz}$ \\
\hline Target response update interval & $1 \mathrm{CPI}$ \\
\hline Number of LFMs in target $-P_{\mathrm{START}}$ & 1 \\
\hline Target start LFM base frequency $-f_{0}$ & $420 \mathrm{MHz}$ \\
\hline Target start LFM bandwidth $-B$ & $60 \mathrm{MHz}$ \\
\hline Number of LFMs in target $-P_{\mathrm{END}}$ & 1 \\
\hline Target end LFM base frequency $-f_{0}$ & $1010 \mathrm{MHz}$ \\
\hline Target end LFM bandwidth $-B$ & $80 \mathrm{MHz}$ \\
\hline Target range & $5 \mathrm{~m}$ \\
\hline Target range profile duration & $384 \mathrm{nS}$ \\
\hline
\end{tabular}

\section{RESULTS AND ANALYSIS}

\section{A. Metrics}

Results from each experimental run, simulation and HITL, are presented in the form of eight data plots. See Fig. 6 for an example. During each experimental run the plots are updated on a CPI basis. Subplots (ad) show a snapshot of data for the current CPI against frequency. Subplots (e-g) show historical results over the course of the experiment. Subplot (h) again shows a snapshot for the current CPI, in this case plotting waveform autocorrelation against time shift (lag).

The contents of each subplot is now described in detail.

Subplot (a) shows the normalised true and estimated instantaneous interference spectrum. The true values, $\mathbf{I}_{\mathrm{PU}}$, are derived directly from the pre-calculated interference table which contains the time domain definitions of the interference waveforms from which $\mathbf{i}$ is the PU waveform for the current processing interval. The estimated data, $\hat{\mathbf{I}}_{\mathrm{PU}}$, is calculated from the noise corrupted data gathered during the environment sensing phase of the experiment, averaged over a number of observations. The estimate can only be updated during the environment sensing phase. For a set of observations, the values are calculated as follows:

$$
\begin{aligned}
\mathbf{I}_{\mathrm{PU}} & =\frac{|\mathscr{F}(\mathbf{i})|}{\max (|\mathscr{F}(\mathbf{i})|)} \\
\hat{\mathbf{I}}_{\mathrm{PU}} & =\frac{\frac{1}{N_{\mathrm{P}}} \sum_{\in N_{\mathrm{P}}}\left|\mathscr{F}\left(\mathbf{r}_{\mathrm{PU}}\right)\right|}{\max \left(\frac{1}{N_{\mathrm{P}}} \sum_{\in N_{\mathrm{P}}}\left|\mathscr{F}\left(\mathbf{r}_{\mathrm{PU}}\right)\right|\right)}
\end{aligned}
$$

where $N_{\mathrm{P}}$ is the number of observations in the assessment, and $\mathbf{r}_{\mathrm{PU}}$ is the received signal from each observation in this phase. Equation (14) represents the actual normalised frequency content of the PU at the current processing interval. Equation (15) calculates the average normalised frequency content of the received PU waveforms.

Subplot (b) illustrates the interrogation waveform, $\mathscr{F}\left(\mathbf{s}_{\mathrm{INT}}\right)$, along with both the mask used to create the notch positions, $\tilde{\mathbf{q}}$, from Section III-A, and the interference estimate from subplot (a) above.

Subplot (c) shows the Fourier domain versions of the signal incident at the target, the pre-defined target response, and the re-radiation of signal from the target. The signal arriving at the target is identical to the interrogation waveform in the simulation case, or, in HITL, its the interrogation waveform subjected to the signal transmission, through the air effects, and the reception process. These plots correspond to Fourier domain versions of (3) and (10), and the $\mathbf{s} * \mathbf{w}$ component of (4) respectively.

Subplot (d) shows the Fourier domain versions of the designed TMI waveform, $\mathbf{S}_{\text {TMI }}$, along with the target response and interference. 


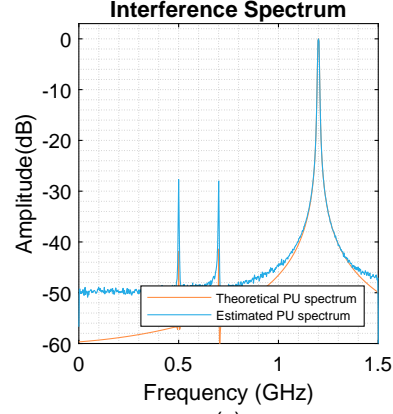

(a)

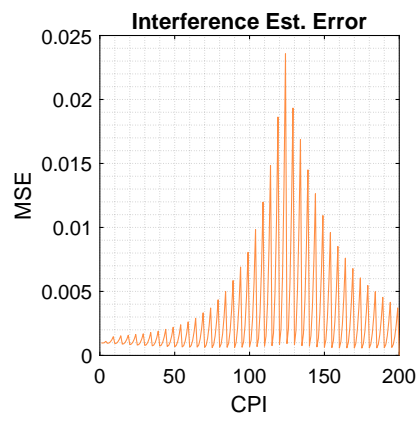

(e)

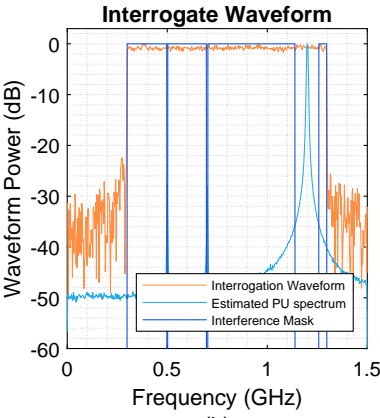

(b)

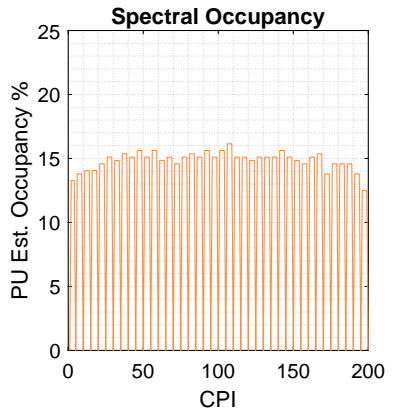

(f)

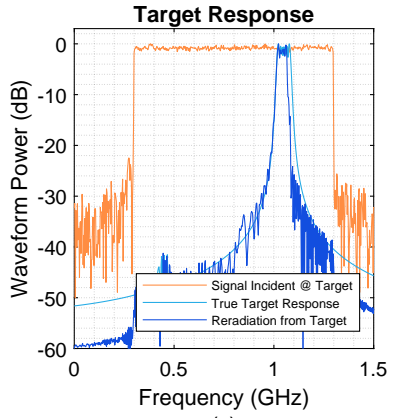

(c)

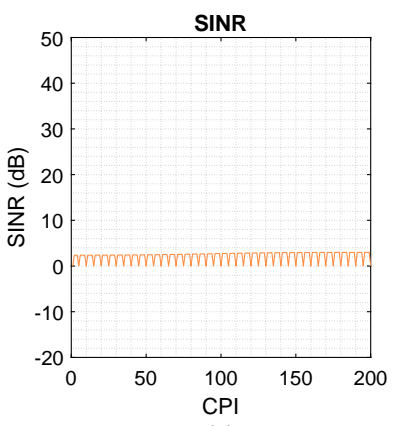

(g)

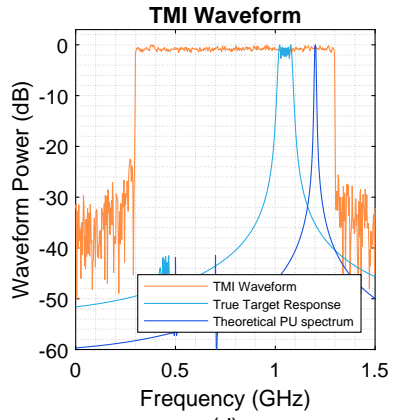

(d)

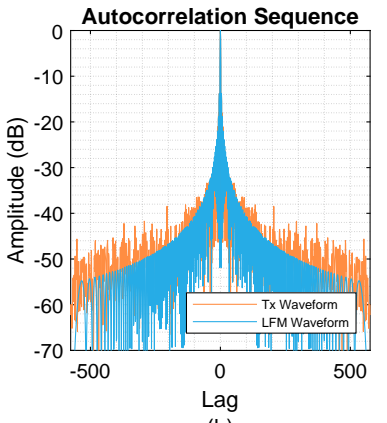

Fig. 6: Performance of non-adaptive waveform in dynamic environment - Simulation. Note the interrogation and TMI waveforms are not modified for environment or target response. The autocorrelation sequence of the transmitted waveform shows good similarity to the basic LFM despite being processed through the ERA.

Subplot (e) shows the sum of the squared estimation error between the estimated interference and the known interference from the interference definition table. The interference estimate can only be calculated during the environmental sensing phase, the error in that estimate approaching zero. During other phases the interference estimate error increases as the actual interference evolves away from the estimated value.

$$
I_{\mathrm{Err}}=\sum_{\in N_{\mathrm{F}}}\left|\mathbf{I}_{\mathrm{PU}}-\hat{\mathbf{I}}_{\mathrm{PU}}\right|^{2}
$$

where $N_{\mathrm{F}}$ is the number of Fourier bins, $\mathbf{I}_{\mathrm{PU}}$ and $\hat{\mathbf{I}}_{\mathrm{PU}}$ are defined by (14) and (15) respectively.

Subplot (f) shows the estimated spectral occupancy of the interference, $\hat{O}$, as estimated by the environment sensing phase processing.

$$
\hat{O}=100 \times\left(1-\frac{\sum_{\in N_{\mathrm{F}}} \tilde{\mathbf{q}}}{N_{\mathrm{F}}}\right)
$$

where $\mathbf{Q}$ is the spectral mask from Section III-A.

Subplot (g) illustrates the estimated SINR for each CPI over the course of the experiment. The estimated
SINR is calculated as:

$$
S I N R=\frac{1}{N_{\mathrm{P}}} \sum_{\in N_{\mathrm{P}}} \frac{\|\mathbf{s} \star \mathbf{r}\|^{2}-X}{X}
$$

where

- $N_{\mathrm{P}}$ is the number of PRIs in the CPI

- $\mathbf{s}$ is the transmit waveform for the CPI

- $\mathbf{r}$ is the received signal collected from each PRI

- $X=\frac{1}{N_{\mathrm{P}}} \sum_{\in N_{\mathrm{P}}}\left\|\mathbf{s} \star \mathbf{r}_{\mathrm{PU}}\right\|^{2}$, the mean of the estimated interference plus noise, $\mathbf{r}_{\mathrm{PU}}$, measured during the environment sensing phase, cross correlated with the current transmit waveform $\mathbf{s}$.

It can be seen in subplots (f-g) the values periodically returning to zero. These correspond to the time region of the environmental sensing phase, where the values are not available.

The final Subplot, (h), provides a comparison of the resolution and sidelobe performance between the ERA processed full band LFM waveform and the designed waveform, by way of their respective autocorrelation sequences.

\section{B. Results}

The following results are based on the radar operating characteristics defined in Table I and the characteristics 
of the interference and target defined in Table II. All parameters apply to both simulation and HITL experiments.

To facilitate the comparison of the FFAST algorithm with non-adaptive, and PU avoidance adaptive techniques, three experimental configuration are considered which employ different waveforms in the exploitation cycle. Namely, a fixed, full bandwidth LFM-like waveform, a notched waveform designed to avoid the PU transmissions, and the FFAST algorithm incorporating TMI. The non-adaptive transmission option is created by setting the spectral mask such that the full bandwidth is available to the radar transmission. The resulting frequency domain representation of the waveform, along with that for the original LFM are shown Fig. 7. It can be seen that the spectral containment at the band edges is sharper for the designed waveform than for the pure LFM. The PU avoidance transmission configuration allows the spectral mask estimated by the MDL to restrict the transmission in areas of PU occupancy, but does not implement TMI. Finally, the FFAST transmission experiment implements the full algorithm.

The subplots (e)-(g) are constructed over the full course of the experiment so are only fully populated at completion. The example plots are snapshots from the end of each experiment.

1) Simulation - non-adaptive transmission: The ERA processed full band LFM waveform case represents a baseline against which the adaptive waveforms may be assessed. Simulation results employing this waveform are shown in Fig. 6. It is clear from subplots (c) and (d) that waveforms with flat frequency content are employed in both the target probe and the TMI phases. The mean SINR attained, excluding the environment sensing phase CPIs, is $2.7 \mathrm{~dB}$. It can be seen in Subplot (h) that the designed transmit waveform exhibits higher sidelobe levels then the original LFM waveform. This increase being due to the non-adaptive waveform being subjected to the same ERA design process as the notched and TMI versions resulting in spectral containment and phase modifications. The sharp spectral cutoff at the required band edges is clearly illustrated in the frequency domain in Fig. 7.

2) Simulation - adaptive notched transmission: The adaptive notched mode uses the notched waveform, created for target interrogation, in the exploitation phase. The target SINR is significantly higher, at a mean of $23 \mathrm{dBs}$, than the non-adaptive case, Fig. $8(\mathrm{~g})$. The autocorrelation sequence is degraded by the inclusion of the notches in terms of sidelobe levels, although resolution is only marginally affected as the waveform continues to exploit the wide bandwidth. It can be seen in Fig. 8 (b) that the notch depth achieved by the ERA is limited to approximately $25 \mathrm{~dB}$.

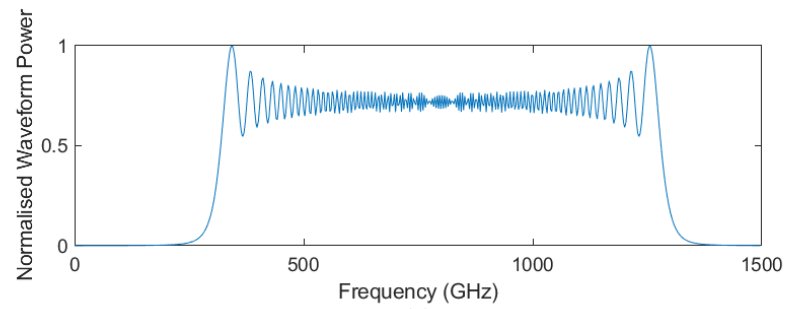

(a)

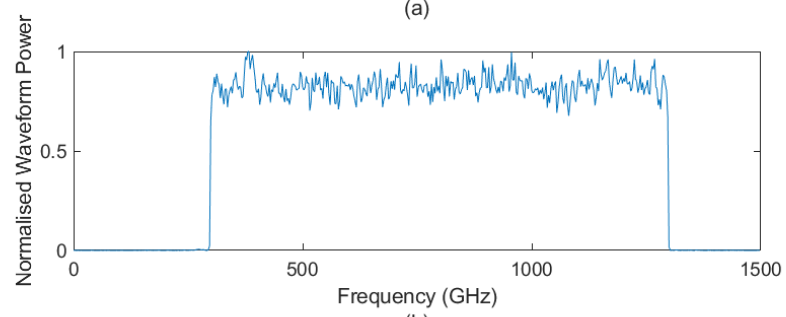

(b)

Fig. 7: Frequency character of (a) the original LFM waveform and (b) the full bandwidth ERA waveform based on the same LFM

3) Simulation - fully adaptive transmission: The TMI waveform created by the FFAST algorithm produces an increase in SINR over the probe waveform, a mean of $40 \mathrm{dBs}$, as the majority of the energy contained in the transmitted signal is concentrated within regions of significant target response. The autocorrelation is degraded in sidelobe terms, but also in resolution as the frequency content of the waveform is significantly reduced, Fig. 9.

4) HITL - non-adaptive transmission: The over-theair transmissions suffer degradation as seen in the signal incident at the target, Fig. 10(c). The SINR is lower, $-6.8 \mathrm{~dB}$, than in the equivalent simulation case. The bandwidth restriction caused by the hardware system can be easily observed in plot (a), where the low and high frequency components of the interference estimate are attenuated by the hardware response.

5) HITL - fully adaptive transmission: Fig. 11 shows results for the full FFAST algorithm with TMI, within the HITL context. The TMI provides an improved SINR of mean $25.5 \mathrm{~dB}$. The autocorrelation sequence suffers both higher sidelobes and decreased resolution.

\section{Discussion}

Comparing the three simulation scenarios, the SINR varies from approximately $3 \mathrm{~dB}$ for the LFM case, to $23 \mathrm{~dB}$ for the notched waveform, up to greater than $40 \mathrm{~dB}$ for FFAST, showing that significant gain is available from the adaptive waveform design process. The disadvantage, in addition to increased computation, and the requirement for flexible transmitting hardware, is in the autocorrelation characteristics of the waveform, 


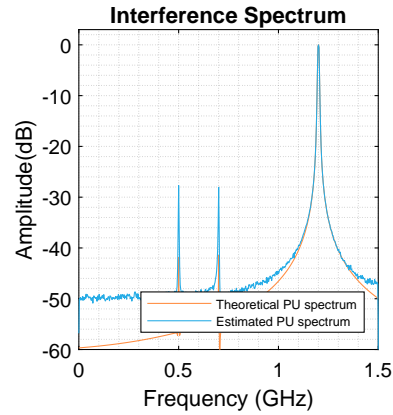

(a)

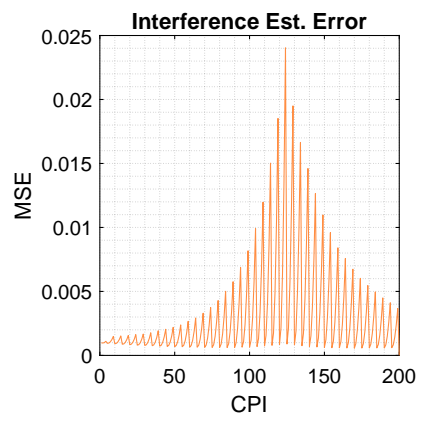

(e)

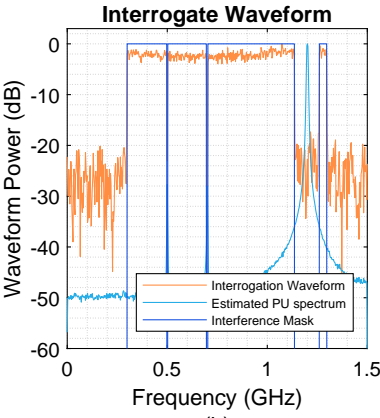

(b)

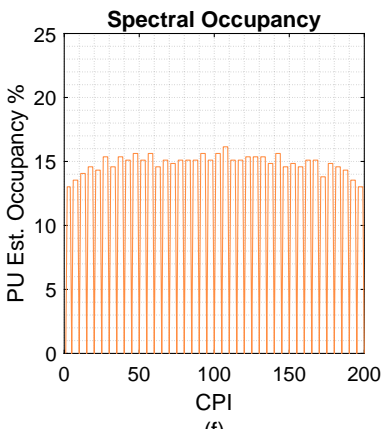

(f)

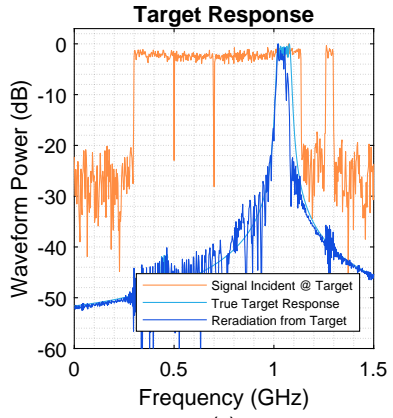

(c)

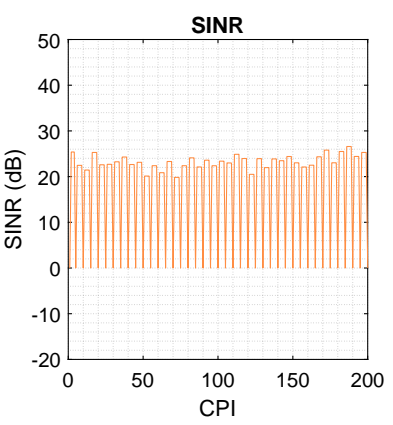

(g)

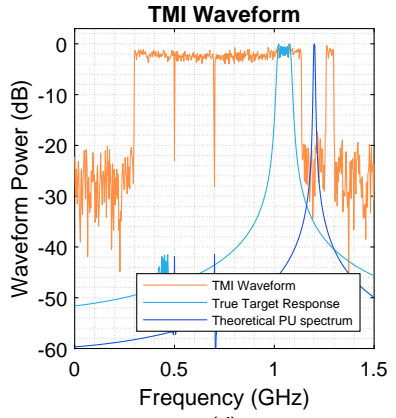

(d)

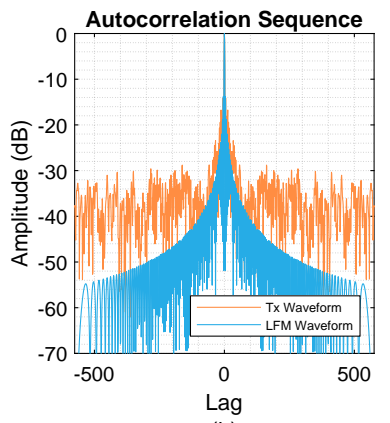

(h)

Fig. 8: Performance of notched LFM waveform in dynamic environment - Simulation. The notch in the interrogation waveform is clearly visible, along with SINR improvement. The inclusion on the waveform notch compromises the autocorrelation sequence side lobe performance.

which exhibits a wider main lobe, resulting in decreased range resolution, and increased sidelobe levels, with the likely consequence of a fall in detection probability or increased false alarm rate. Table III summarises the autocorrelation characteristics for a single snapshot of the ERA processed LFM, the notched LFM and the TMI signals, taken from the final CPI of each simulation run. Each result is normalised to the LFM value. Although the peak sidelobe level remains fairly constant, the integrated sidelobes increase as the waveform design becomes more specialised. The $3 \mathrm{~dB}$ width of the main lobe of the autocorrelation is stable between the LFM and notched waveforms, but is significantly degraded in the TMI case.

The SINR for the LFM is reduced to approximately $-6 \mathrm{~dB}$ in the HITL scenario. A drop of $9 \mathrm{~dB}$ from the simulation case. For TMI, the SINR decrease between simulation and HITL is from approximately $40 \mathrm{~dB}$ to 30 $\mathrm{dB}$. The decrease in SINR is not unexpected due to that fact the hardware aspects and physical effects, such as range attenuation, were not modelled in the simulation cases.

Fig. 12 summarises the SINR results for the three waveform cases over the duration of the experiments for (a) simulation and (b) HITL cases. In the simulation case it can be seen that the LFM provides almost constant SINR over all CPIs. The notched waveform rejects the PU interference to gain approximately 20 $\mathrm{dBs}$ of SINR. The results from the target matched waveform provides an additional $20 \mathrm{dBs}$ of SINR, but with significant variability between CPIs. As expected the HITL results provide reduced SINR over the three waveforms as transmission/reception effects are not included in the simulation results. However, similar gains are observed across the waveforms, although individual values are more variable. It should be stressed that the gain achieved by FFAST is at the cost of reduced range resolution. The zero values seen periodically on the plots are due to target SINR being unavailable during the environmental sensing phase.

The results of an assessment of the effects of PU occupancy extent on SINR can be seen in Fig. 13. The target is defined to exist outside of the PU occupancy. Identical total interference power is employed across the occupancy bandwidth in all cases. The SINR result for the non-adaptive waveform is constant over the range of occupancy extent. The adaptive notched waveform, benefits from increasing PU occupancy. This is to be expected as the designed waveform is normalised such that constant power is transmitted independent of the bandwidth covered. This results in the transmit power 


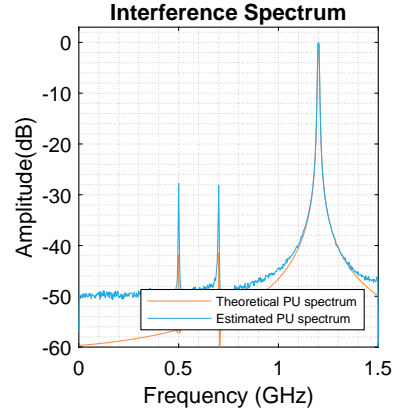

(a)

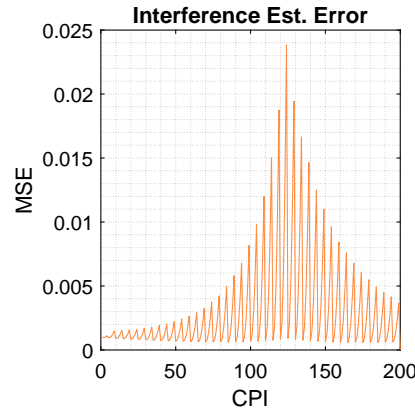

(e)

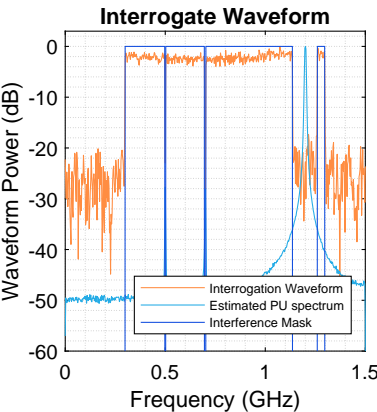

(b)

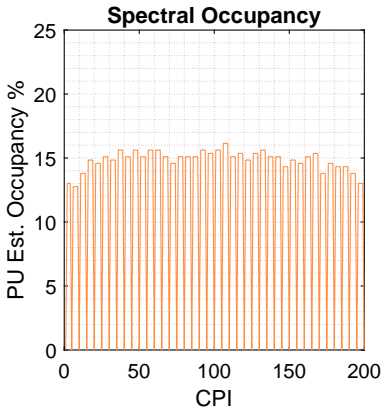

(f)

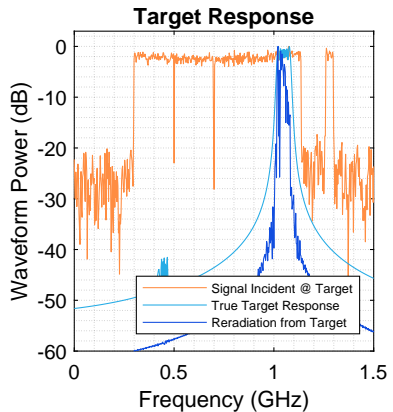

(c)

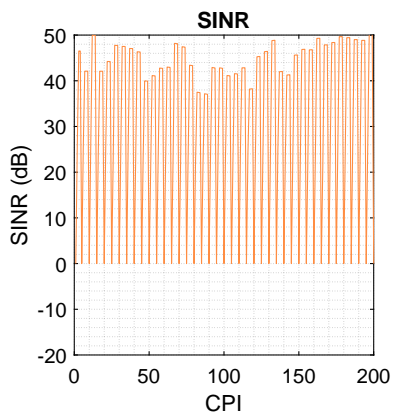

(g)

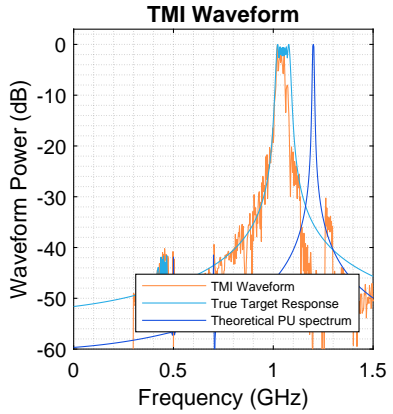

(d)

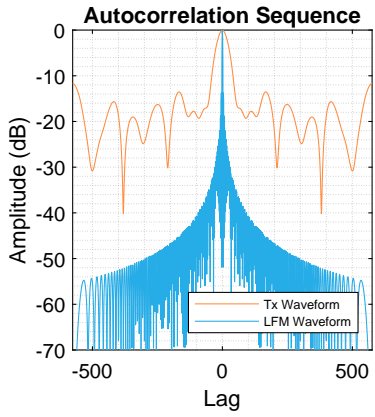

(h)

Fig. 9: Performance of FFAST algorithm in dynamic environment - Simulation. The TMI waveform closely resembles the target response providing further SINR improvement over the notched waveform, at a cost of degraded main lobe width and side lobe levels in the autocorrelation sequence.

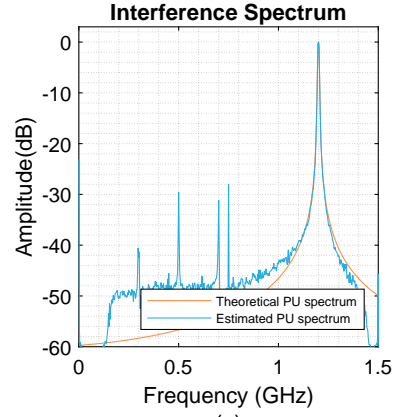

(a)

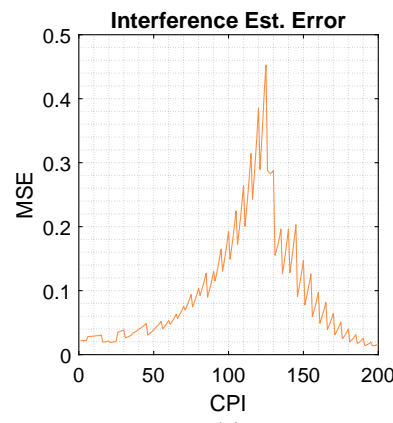

(e)

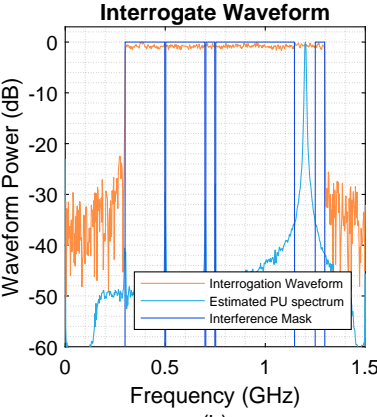

(b)

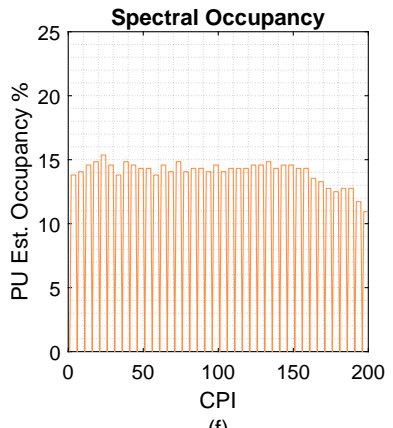

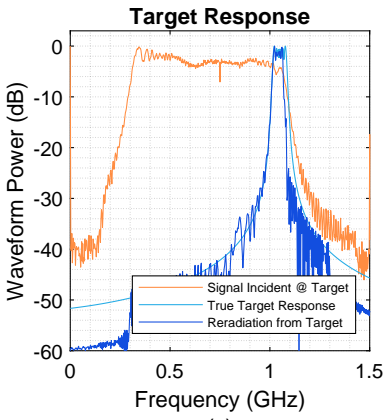

(c)

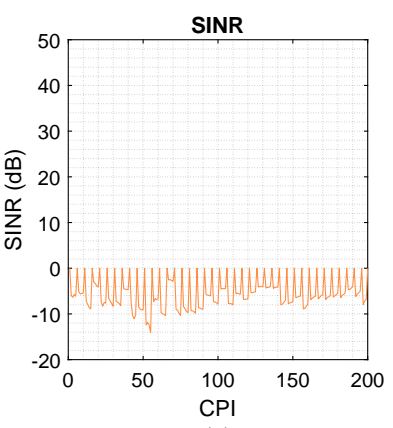

(g)

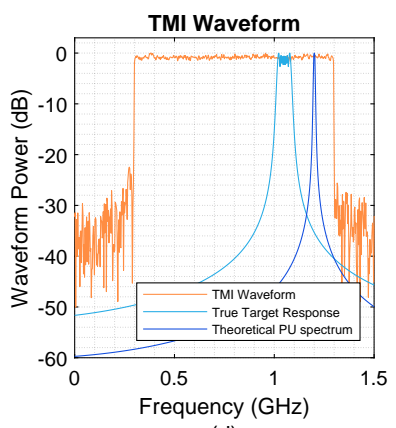

(d)

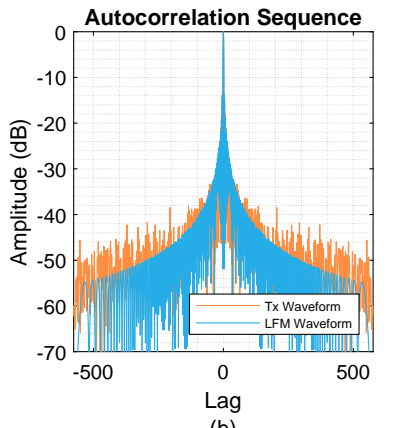

(h)

Fig. 10: Performance of non-adaptive waveform in dynamic environment - HITL. Of note is the reduction and greater variability in SINR compared with the simulation results. 
TABLE III: Waveform Autocorrelation Results Normalised to the LFM

\begin{tabular}{|c|c|c|c|c|}
\cline { 2 - 5 } \multicolumn{1}{c|}{} & \multicolumn{4}{c|}{ Waveform type } \\
\hline Characteristic & LFM & LFM-ERA & Notched & TMI \\
\hline Peak Sidelobe Levels (dB) & 0 & 0 & -0.3 & -0.9 \\
\hline Integrated Sidelobe Levels (dB) & 0 & 1.0 & 6.5 & 13.6 \\
\hline Mainlobe (3dB) Width & 1.0 & 1.1 & 1.1 & 16.4 \\
\hline
\end{tabular}

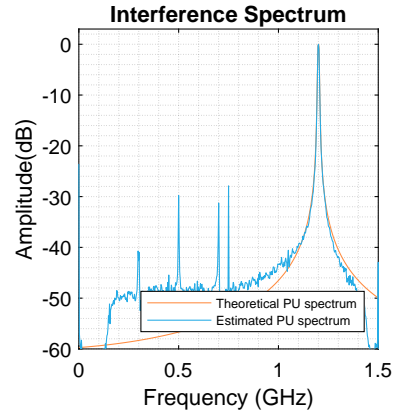

(a)

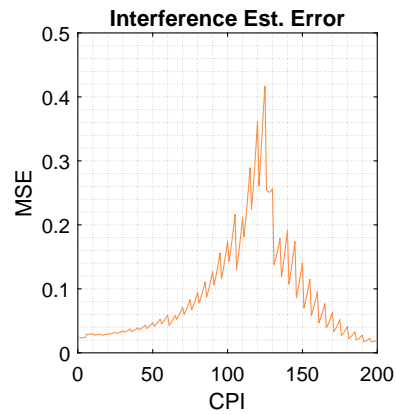

(e)

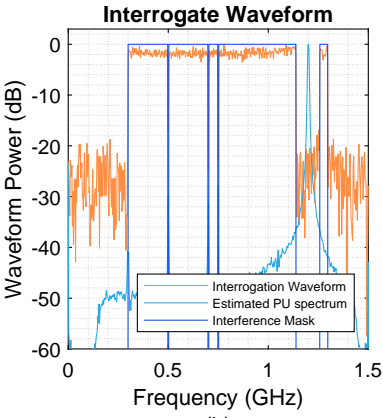

(b)

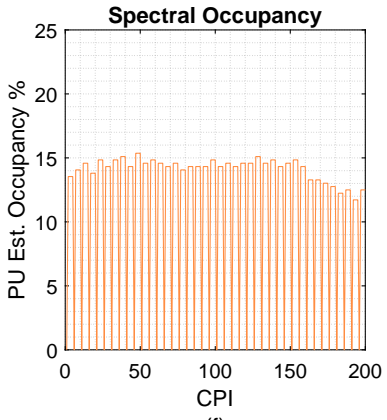

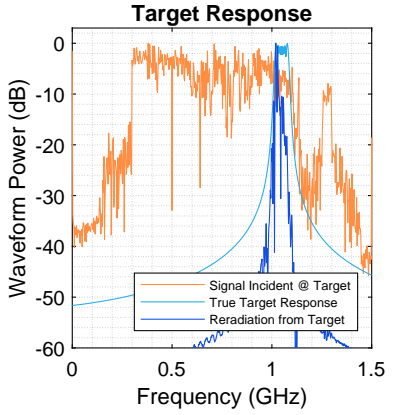

(c)

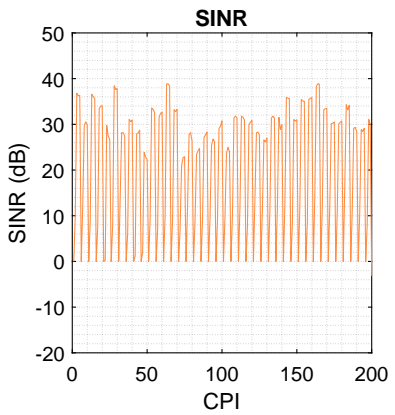

(g)

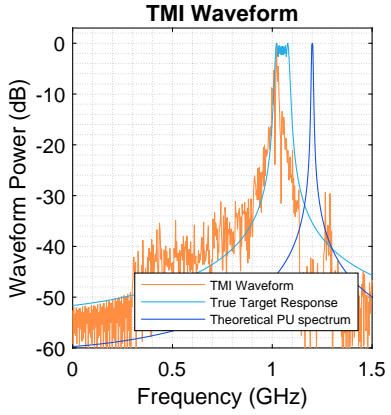

(d)

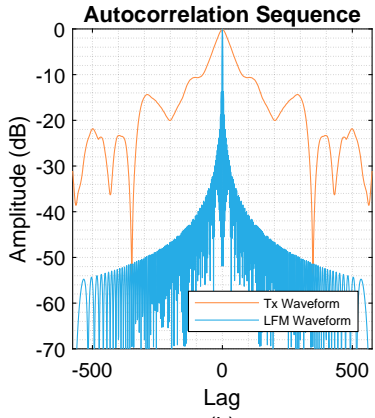

(h)

Fig. 11: Performance of FFAST algorithm in dynamic environment - HITL. Transmitting the TMI waveform overthe-air is seen to result in a significant rise in SINR showing that the waveform design techniques can be applied in practical systems.

being more concentrated in the region where the target exists as the PU occupancy increases, resulting in the superior SINR. The FFAST algorithm achieves additional gains in SINR as the available power is further focused on the target occupancy region. The SINR is relatively constant with PU occupancy as the power is always directed towards the target response, irrespective of PU occupancy.

\section{CONCLUSION}

In this work we describe simulation and practical experimentation investigating the use of a closed loop processing architecture for the spectral coexistence of a cognitive radar with a spectrally dynamic primary user of the RF spectrum. The scenario requires the implementation of environmental sensing and waveform design based on the perception of environmental and target characteristics. The problem is further constrained by requiring a phase only design technique, and critically, low computational cost.

Significant SINR gains have been demonstrated in both simulation and hardware-in-the-loop regimes, with notched waveforms providing approximately $20 \mathrm{~dB}$ of SINR gain over the non-adaptive case. The same algorithm has been applied to perform TMI, which provides in excess of $20 \mathrm{~dB}$ of additional gain.

The framework employed allows repeatable testing when using hardware-in-the-loop, by simulating both the primary user interference signal, and the target using components of a flexible experimental radar system.

The cost of the additional gain, in addition to the extra processing load to assess the interference and to design the waveform, can be seen in the degradation in the autocorrelation performance of the PU avoidance 


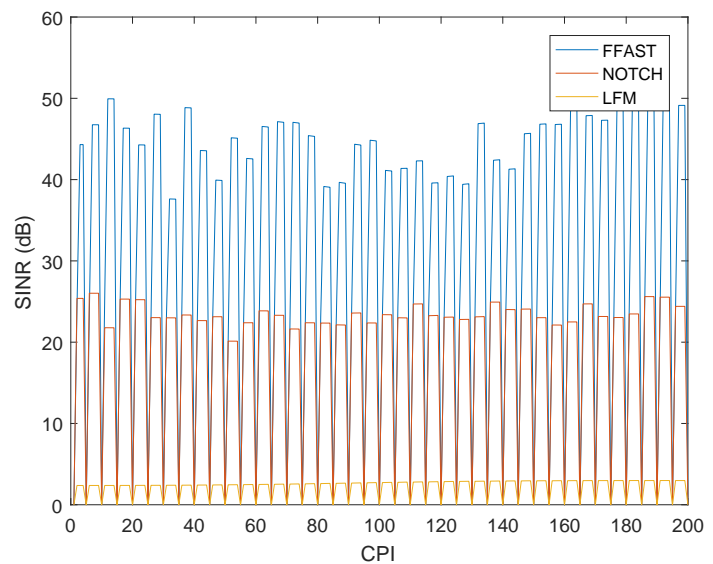

(a)

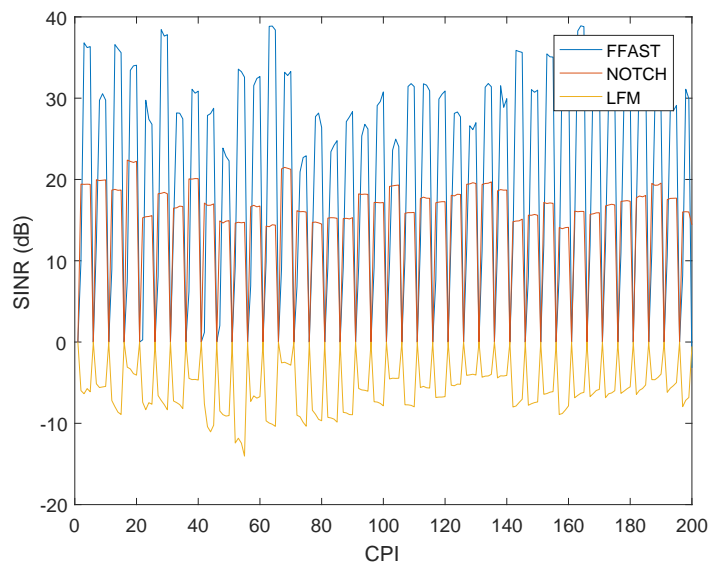

(b)

Fig. 12: Comparison of SINR over 200 CPIs for LFM, Notched and FFAST waveforms for (a) Simulation and (b) HITL experiments. The range extent of each plot is $60 \mathrm{~dB}$, offset by $-20 \mathrm{~dB}$ between the simulated and HITL results. Similar trends of increasing SINR are seen across the three waveform types in both the simulation and HITL cases.

and target matched waveforms. For the example case analysed, the peak sidelobes show small improvements for the Notched and TMI cases. The integrated sidelobe levels degrade by $6.5 \mathrm{~dB}$ and $13.6 \mathrm{~dB}$ for the Notched and TMI waveforms respectively. The mainlobe width for the TMI is over 16 times greater for the TMI waveform as compared with the other three. Future work should consider the application of additional constraints to improve sidelobe performance and range resolution.

The $25 \mathrm{~dB}$ notch depth achieved by the ERA is shallow compared to other known methods, such as Reiterative Uniform Weight Optimization (RUWO) [65]. Future work should therefore consider employing RUWO or

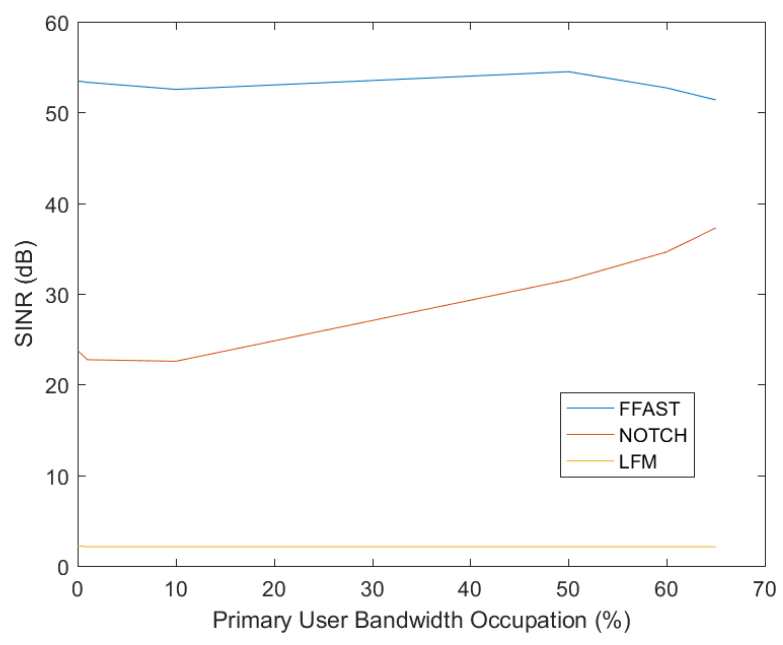

Fig. 13: Comparison of SINR against PU spectral occupation for LFM, Notched and FFAST waveforms. The percentage PU occupancy shows little effect on the FFAST waveform performance assuming the target response is outside of the occupancy region.

similar methods within the framework presented here, with the aim of increased notch depth. In addition, the replacement of the simulated target with a physical target model, placed on a turntable would provide further evidence of the gain achievable.

\section{ACKNOWLEDGMENTS}

The authors acknowledge the support of EPSRC and the Defence Science and Technology Laboratory (Dstl). The authors also wish to acknowledge the support of $\mathrm{Dr}$ Adam Mitchell, Mr John-Peter Baptiste, Mr Jakob Long and Mr Richard Walkerdine during the data collection.

Lastly, this work was supported by the Air Force Office of Scientific Research. However, the views and opinions expressed in this article are those of the authors and do not necessarily reflect the official policy or position of any agency of the U.S. government. Examples of analysis performed within this article are only examples. Assumptions made within the analysis are also not reflective of the position of any U.S. Government entity.

\section{REFERENCES}

[1] M. Wicks, "Spectrum crowding and cognitive radar," in 2nd International Workshop on Cognitive Information Processing. 1416 June, 2010, (Elba Island), pp. 452-457, 2010.

[2] H. Griffiths, S. Blunt, L. Cohen, and L. Savy, "Challenge problems in spectrum engineering and waveform diversity," in IEEE Radar Conference. 29 April-3 May, 2013, (Ottawa), 2013.

[3] R. Gomez-Garcia, J. P. Magalhaes, J. M. Munoz-Ferreras, J. M. N. Vieira, N. B. Carvalho, and J. Pawlan, "Filling the spectral holes," IEEE Microwave Magazine, vol. 15, no. 2, pp. 45-56, 2014. 
[4] H. Griffiths, L. Cohen, S. Watts, E. Mokole, C. Baker, M. Wicks, and S. Blunt, "Radar spectrum engineering and management: Technical and regulatory issues," Proc. IEEE, vol. 103, no. 1, pp. 85-102, 2015.

[5] UK Department for Culture Media \& Sport, "Enabling UK growth - Releasing public spectrum. Making $500 \mathrm{MHz}$ of spectrum available by 2020," Tech. Rep. March, 2011.

[6] UK Department for Culture Media \& Sport, "The UK spectrum strategy. Delivering the best value from spectrum for the UK," Tech. Rep. March, 2014.

[7] Federal Communications Commission, "Auction of advanced wireless services (Aws-3) licenses closes," 2015.

[8] UK Ministry of Defence, "Strategic trends programme. Future operating environment 2035. First edition," tech. rep., 2015.

[9] S. D. Blunt and E. Perrins, eds., Radar and Communication Spectrum Sharing. London: Scitech Publishing, 2018.

[10] S. Haykin, "Cognitive radar [A way of the future]," IEEE Signal Processing Magazine, vol. 23, no. January, pp. 30-40, 2006.

[11] J. R. Guerci, "Cognitive radar : The next radar wave?" Microwave Journal, vol. 54, no. 1, pp. 22-36, 2011.

[12] M. S. Greco, F. Gini, P. Stinco, and K. Bell, "Cognitive radars: On the road to reality: Progress thus far and possibilities for the future," IEEE Signal Processing Magazine, vol. 35, no. 4, pp. 112-125, 2018.

[13] A. M. Jones, C. P. Horne, H. D. Griffiths, G. Smith, A. Mitchell, and P. John-Baptiste, "Experimention of an Adaptive \& Autonomous RF Signaling Strategy For Detection," in IEEE Radar Conference, (Oklahoma), 2018.

[14] J. W. Garnham and J. R. Roman, "Why and what is waveform diversity, and how does it affect electromagnetics?" in IEEE International Symposium on Electromagnetic Compatibility. 9-13 July, 2007, (Honolulu), 2007.

[15] M. C. Wicks, "A brief history of waveform diversity," in IEEE Radar Conference. 4-8 May, 2009, (Passadena), 2009.

[16] S. U. Pillai and M. E. Davis, "Waveform diversity for ultra-wide band surveillance radars," IET Radar, Sonar \& Navigation, vol. 8, no. 9 , pp. 1226-1233, 2014

[17] S. D. Blunt and E. L. Mokole, "An overview of radar waveform diversity," IEEE Aerospace \& Electronic System Magazine, vol. 31, no. 11, pp. 2-40, 2016.

[18] S. Haykin, Y. Xue, and T. N. Davidson, "Optimal waveform design for cognitive radar," in Asilomar Conference on Signals, Systems and Computers. 26-29 Oct., 2008, (Pacific Grove), pp. 37, 2008.

[19] M. R. Bell, "Information theory and radar waveform design," IEEE Transactions on Information Theory, vol. 39, no. 5, pp. 1578-1597, 1993.

[20] P. Setlur, N. Devroye, and M. Rangaswamy, "Radar waveform design with the two step mutual information," IEEE National Radar Conference - Proceedings, pp. 1317-1322, 2014.

[21] M. Piezzo, A. De Maio, A. Aubry, and A. Farina, "Cognitive radar waveform design for spectral coexistence," in IEEE Radar Conference. 29 April-3 May, 2013, (Ottawa), 2013.

[22] I. W. Selesnick, S. U. Pillai, and R. Zheng, "An iterative algorithm for the construction of notched chirp signals," in IEEE Radar Conference. 10-14 May, 2010, (Washington, DC), pp. 200 203, 2010.

[23] I. W. Selesnick and S. U. Pillai, "Chirp-like transmit waveforms with multiple frequency-notches," in IEEE Radar Conference. 23-27 May, 2011, (Kansas City), pp. 1106-1110, 2011.

[24] B. Ravenscroft, S. D. Blunt, C. Allen, A. Martone, and K. Sherbondy, "Analysis of spectral notching in FM noise radar using measured interference," in IET Radar Conference. 23-26 Oct. 2017, (Belfast), 2017.

[25] J. W. Owen, B. Ravenscroft, B. H. Kirk, S. D. Blunt, C. T. Allen, A. F. Martone, K. D. Sherbondy, and R. M. Narayanan, "Experimental demonstration of cognitive spectrum sensing \& notching for radar," in IEEE Radar Conference. 23-27 April, 2018, (Oklahoma), 2018.

[26] A. Aubry, V. Carotenuto, A. De Maio, A. Farina, and L. Pallotta, "Optimization theory-based radar waveform design for spectrally dense environments," IEEE Aerospace and Electronic Systems Magazine, vol. 31, no. 12, pp. 14-25, 2016.
[27] Y. Huang, M. Piezzo, V. Carotenuto, and A. De Maio, "Radar waveform design under similarity, bandwidth priority, and spectral coexistence constraints," in 2017 IEEE Radar Conference, (Seattle), pp. 1142-1147, IEEE, 2017.

[28] S. W. Frost and B. Rigling, "Sidelobe predictions for spectrallydisjoint radar waveforms," in IEEE Radar Conference. 7-11 May, 2012, (Atlanta, GA), pp. 0247-0252, 2012.

[29] M. La Manna, P. Stinco, M. Greco, and F. Gini, "Design of a cognitive radar for operation in spectrally dense environments," in IEEE Radar Conference. 29 April-3 May, 2013, (Ottawa), 2013.

[30] D. T. Gjessing, Target Adaptive Matched Illumination Radar: Principles \& Applications. London: Peter Peregrinus Ltd, 1986.

[31] J. R. Guerci and S. Pillai, "Theory and application of optimum transmit-receive radar," in IEEE International Radar Conference. 7-12 May, 2000, (Alexandria), pp. 705-710, 2000.

[32] D. A. Garren, M. K. Osborn, A. C. Odom, J. S. Goldstein, S. U. Pillai, and J. R. Guerci, "Enhanced target detection and identification via optimised radar transmission pulse shape," IET Radar, Sonar and Navigation, vol. 148, no. 3, pp. 130-138, 2001.

[33] V. Karimi, R. Mohseni, and S. Samadi, "Adaptive OFDM Waveform Design for Cognitive Radar in Signal-Dependent Clutter," IEEE Systems Journal, pp. 1-11, 2019.

[34] X. Yu, G. Cui, P. Ge, and L. Kong, "Constrained radar waveform design algorithm for spectral coexistence," Electronics Letters, vol. 53, no. 8, pp. 558-560, 2017.

[35] R. Palamà, H. Griffiths, and F. Watson, "A radar architecture for joint dynamic spectrum access and target-matched illumination," in IEEE Radar Conference. 8-12 May, 2017, (Seattle), pp. 14721477, 2017.

[36] L. Bennett, S. Member, J. P. Toomey, and S. Member, "Target Classification with Multiple Frequency Illumination," IEEE Transactions on Antennas and Propagation, vol. 29, no. 2, pp. $352-358,1981$.

[37] D. A. Garren, A. C. Odom, M. K. Osborn, J. S. Goldstein, S. U. Pillai, and J. R. Guerci, "Full-polarization matched-illumination for target detection and identification," IEEE Transactions on Aerospace and Electronic Systems, vol. 38, no. 3, pp. 824-837, 2002.

[38] N. A. Goodman, P. R. Venkata, and M. A. Neifeld, "Adaptive waveform design and sequential hypothesis testing for target recognition with active sensors," IEEE Journal on Selected Topics in Signal Processing, vol. 1, no. 1, pp. 105-113, 2007.

[39] J. Bae and N. A. Goodman, "Evaluation of modulus-constrained matched illumination waveforms for target identification," in IEEE Radar Conference. 10-14 May, 2010, (Washington), pp. 871-876, 2010.

[40] A. Aubry, A. D. Maio, M. Piezzo, M. M. Naghsh, M. Soltanalian, and P. Stoica, "Cognitive radar waveform design for spectral coexistence in signal-dependent interference," in IEEE Radar Conference. 19-23 May, 2014, (Cincinnati), pp. 474-478, 2014.

[41] X. Zhang, K. Wang, and X. Liu, "Joint optimisation of transmit waveform and receive filter for cognitive radar," IET Radar, Sonar \& Navigation, vol. 12, no. 1, pp. 11-20, 2018.

[42] B. H. Kirk, J. W. Owen, R. M. Narayanan, S. D. Blunt, A. F. Martone, and K. D. Sherbondy, "Cognitive software defined radar: waveform design for clutter and interference suppression," Radar Sensor Technology XXI, vol. 10188, no. May 2017, p. 1018818, 2017.

[43] R. Romero and N. Goodman, "Waveform design in signaldependent interference and application to target recognition with multiple transmissions," IET Radar, Sonar \& Navigation, vol. 3, no. 4, pp. 328-340, 2009.

[44] R. A. Romero, J. Bae, and N. A. Goodman, "Theory and application of SNR and mutual information matched illumination waveforms," IEEE Transactions on Aerospace and Electronic Systems, vol. 47, no. 2, pp. 912-927, 2011.

[45] L. K. Patton and B. D. Rigling, "Modulus constraints in adaptive radar waveform design," in IEEE Radar Conference, (Rome), pp. 1-6, 2008.

[46] L. Patton, S. Frost, and B. Rigling, "Efficient design of radar waveforms for optimised detection in coloured noise," IET Radar, Sonar \& Navigation, vol. 6, no. 1, p. 21, 2012. 
[47] J. De Graaf, H. Faust, J. Alatishe, and S. Talapatra, "Generation of spectrally confined transmitted radar waveforms: experimental results," in IEEE Radar Conference. 24-27 April, 2006, (Verona, NY), pp. 76-83, 2006.

[48] A. Aubry, A. De Maio, M. Piezzo, and A. Farina, "Radar waveform design in a spectrally crowded environment via nonconvex quadratic optimization," IEEE Transactions on Aerospace and Electronic Systems, vol. 50, no. 2, pp. 1138-1152, 2014.

[49] C. Baylis, M. Fellows, L. Cohen, and R. J. Marks, "Solving the spectrum crisis: Intelligent, reconfigurable microwave transmitter amplifiers for cognitive radar," IEEE Microwave Magazine, vol. 15 , no. 5, pp. 94-107, 2014.

[50] A. Aubry, V. Carotenuto, A. D. Maio, and S. Iommelli, "Cognitive radarwWaveform design for spectral compatibility," in Sensor Signal Processing for Defence. 22-23 Sept., 2016, (Edinburgh), 2016.

[51] M. Yli-Niemi and S. A. Vorobyov, "Computationally efficient waveform design in spectrally dense environment," Proceedings of the IEEE Sensor Array and Multichannel Signal Processing Workshop, vol. 2018-July, pp. 277-281, 2018.

[52] S. Bruggenwirth, "Design and implementation of a three-layer cognitive radar architecture," in Asilomar Conference on Signals, Systems and Computers. 6-9 Nov., 2016, (Pacific Grove), pp. 929933, 2016.

[53] Y. Zeng and Y. C. Liang, "Spectrum-sensing algorithms for cognitive radio based on statistical covariances," IEEE Transactions on Vehicular Technology, vol. 58, no. 4, pp. 1804-1815, 2009.

[54] P. Stinco, M. S. Greco, and F. Gini, "Spectrum sensing and sharing for cognitive radars," IET Radar, Sonar \& Navigation, vol. 10, pp. 595-602, 2016.

[55] Pu Wang, Jun Fang, Ning Han, and Hongbin Li, "MultiantennaAssisted Spectrum Sensing for Cognitive Radio," IEEE Transactions on Vehicular Technology, vol. 59, no. 4, pp. 1791-1800, 2009.

[56] A. Aubry, V. Carotenuto, A. De Maio, and M. A. Govoni, "MultiSnapshot Spectrum Sensing for Cognitive Radar via BlockSparsity Exploitation," IEEE Transactions on Signal Processing, vol. 67, no. 6, pp. 1396-1406, 2019.

[57] M. Wax and T. Kailath, "Detection of signals by information theoretic criteria," IEEE Transactions on Acoustics, Speech, and Signal Processing, vol. 33, no. 2, pp. 387-392, 1985.

[58] P. Stoica and Y. Selén, "Model-Order Selection," IEEE Signal Processing Magazine, vol. 21, no. 4, pp. 36-47, 2004.

[59] C. L. Costa, A. Thakre, F. Roemer, M. Haardt, P. O. Box, and D. Ilmenau, "Comparison of model order selection techniques for high-resolution parameter estimation algorithms," in Internationales Wissenschaftliches Kolloquium, (Ilmenau), 2009.

[60] R. Gerchberg and W. Saxton, "A practical approach for the determination of the phase from image and diffraction plane pictures," Optik, vol. 237-246, 1972.

[61] J. R. Fienup, "Phase retrieval algorithms: a comparison," Applied Optics, vol. 21, no. 15, p. 2758, 1982.

[62] A. De Maio and A. Farina, "Waveform diversity: Past, present, and future," in RTO-EN-SET-119(2009), 2009.

[63] S. De Nicola, Y. Huang, A. De Maio, S. Zhang, and A. Farina, "Code optimization with similarity and accuracy constraints," in IEEE Radar Conference. 26-30 May, 2008, (Rome), 2008.

[64] G. E. Smith, Z. Cammenga, A. Mitchell, K. L. Bell, J. Johnson, M. Rangaswamy, and C. Baker, "Experiments with cognitive radar," IEEE Aerospace and Electronic Systems Magazine, vol. 31, no. 12, pp. 34-46, 2016.

[65] T. Higgins, T. Webster, and A. K. Shackelford, "Mitigating interference via spatial and spectral nulling: Open air experimental results," IET Radar, Sonar and Navigation, vol. 8, no. 2, pp. 8493, 2014. 